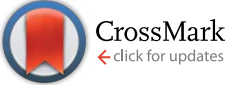

Cite this: RSC Adv., 2017, 7, 9704

\title{
Syntheses, structural diversities and characterization of a series of coordination polymers with two isomeric oxadiazol-pyridine ligands $\uparrow$
}

\author{
Bin Ding, ${ }^{\star a b}$ Jie Wu, ${ }^{\text {ab }}$ Xiang Xia Wu, ${ }^{\text {ab }}$ Jian Zhong Huo, ${ }^{\text {ab }}$ Zhao Zhou Zhu, ${ }^{\text {ab }}$ \\ Yuan Yuan Liu ${ }^{\mathrm{ab}}$ and Fang Xue Shi ${ }^{\mathrm{ab}}$
}

In this work two positional-isomeric oxadiazol-pyridine ligands 3-(5-methyl-1,3,4-oxadiazol-2-yl)pyridine $\left(L_{1}\right)$ and 4-(5-methyl-1,3,4-oxadiazol-2-yl)pyridine $\left(L_{2}\right)$ have been designed and synthesized. A series of novel coordination polymers, namely $\left[\mathrm{Cd}_{2}\left(\mu_{2}-\mathrm{L}_{1}\right)_{2}\left(\mu_{2}-\mathrm{NCS}\right)_{4}\right]_{n}(1),\left\{\left[\mathrm{Cd}\left(\mathrm{L}_{1}\right)\left(\mu_{2}-\mathrm{dca}\right)_{2}\left(\mathrm{H}_{2} \mathrm{O}\right)\right] \cdot \mathrm{H}_{2} \mathrm{O}\right\}_{n} \quad(2)$, $\left\{\left[\mathrm{Cu}\left(\mu_{2}-\mathrm{L}_{1}\right)_{2}(\mathrm{NCS})_{2}\right] \cdot 0.5 \mathrm{H}_{2} \mathrm{O}\right\}_{n} \quad(3), \quad\left\{\left[\mathrm{Ag}_{2}\left(\mu_{2}-\mathrm{L}_{1}\right)\left(\mu_{3}-\mathrm{L}_{1}\right)_{2}\right] \cdot 2 \mathrm{PF}_{6}\right\}_{n} \quad(4), \quad\left\{\left[\mathrm{Ag}_{3}\left(\mu_{2}-\mathrm{L}_{1}\right)_{4}\left(\mu_{2}-\mathrm{CF}_{3} \mathrm{SO}_{3}\right)\left(\mathrm{CF}_{3} \mathrm{SO}_{3}\right)\right]\right.$. $\left.\mathrm{CF}_{3} \mathrm{SO}_{3}\right\}$ (5), $\left\{\left[\mathrm{Cd}\left(\mathrm{L}_{2}\right)_{2}\left(\mu_{2}-\mathrm{NCS}\right)_{2}\right]\right\}_{n}(6),\left[\mathrm{Ag}\left(\mu_{2}-\mathrm{L}_{2}\right)\left(\mu_{2}-\mathrm{CF}_{3} \mathrm{SO}_{3}\right)\right]_{n}(7)$ and $\left\{\left[\mathrm{Ag}\left(\mu_{2}-\mathrm{L}_{2}\right)\right] \cdot \mathrm{BF}_{4}\right\}_{n}$ (8) have been isolated. Both 1 and 2 are 2D Cd" coordination polymers containing infinite $\{\mathrm{Cd}-\mathrm{NCS}-\mathrm{Cd}$ \} chains (for 1 ) or infinite $\{\mathrm{Cd}-\mathrm{dca}-\mathrm{Cd}\}$ layers (for 2 ), respectively. 3 is a $2 \mathrm{D} \mathrm{Cu}$ " coordination polymer, in which central metal ions are bridged via a bidentate bridging $\mathrm{L}_{1}$ ligand. While when different $\mathrm{Ag}^{\prime}$ salts were introduced into the reaction system, 1D Ag' coordination polymers 4 and 5 with diverse coordination modes can be isolated. Furthermore, when the isomeric oxadiazol-pyridine $L_{2}$ is used to replace $L_{1}$ in the reaction system, $6-8$ can be isolated. 6 is a 2D Cd" coordination polymer containing $\{\mathrm{Cd}-\mathrm{NCS}-\mathrm{Cd}$ \} layers. 7 is a $2 \mathrm{D}$ neutral $\mathrm{Ag}^{\prime}$ coordination polymer while 8 is a $2 \mathrm{D}$ cationic $\mathrm{Ag}^{\prime}$ coordination polymer. Variable temperature magnetic susceptibility measurements $(2-300 \mathrm{~K})$ reveal anti-ferromagnetic interactions between central copper(II) ions for 3 . Solid-state luminescent properties of 1, 2 and 4-8 have been investigated indicating strong fluorescent emissions. Additionally, luminescent measurements illustrate that complex 8 exhibits highly sensitive luminescence sensing for $\mathrm{Cr}_{2} \mathrm{O}_{7}{ }^{2-}$ ions in aqueous solutions with high quenching efficiency $K_{\text {sv }}=2.08 \times 10^{4} \mathrm{~L} \mathrm{~mol}^{-1}$ and low detection limit $(0.19 \mu \mathrm{M}(\mathrm{S} / \mathrm{N}=3)) .8$ also represents the first report of a coordination polymer based on oxadiazol-pyridine derivatives with a luminescence response to $\mathrm{Cr}_{2} \mathrm{O}_{7}^{2-}$ anion pollutants in aqueous solutions.

Received 13th December 2016 Accepted 27th January 2017

DOI: $10.1039 / c 6 r a 28153 b$

rsc.li/rsc-advances be applied in the selective and sensitive detection of environmental pollutants, which can further protect human health and the environment from harm by these pollutants. ${ }^{10}$ Their tunable channel size, good structural stability, and large surface area of luminescent coordination polymers make these coordination materials more competitive over other luminescent materials because more analytes can effectively interact with the channel surface areas of the coordination polymers, which can ultimately improve the rate of luminescence response, decreasing the fluorescence detection limit and improving the detection sensitivity. ${ }^{11}$

In the crystal engineering of functional coordination polymers, the variable selection of metal ions and organic ligands provides endless possible outcomes of the final products. Although these porous coordination polymers with targeted secondary building units (SBUs) and topologies can be synthesized by careful selection of the metal ions and organic ligands, the final structures of the products are still highly influenced by several factors including metal-ligand ratio, $\mathrm{pH}$, solvent, 
temperature, and soon. In particular, judicious selection of ligands is important for deliberate structural changes and functional properties of coordination polymers. ${ }^{12}$ Therefore many new flexible or rigid ligands have been explored and assembled into metal-organic polymers via coordination bonds or supramolecular interactions such as hydrogen bonds, $\pi-\pi$ stacking, and host-guest ionic interactions et al. ${ }^{\mathbf{1 3 - 1 6}}$

1,3,4-Oxidazoles can be employed as one kind of efficient bridging ligands and offer a range of charge-balance requirements, alternative linking modes and different orientations of donor groups. ${ }^{17,18}$ Currently oxidazole-pyridine and their derivatives have gained more interest as ligands to bridge different metal ions to form functional coordination polymers because of their intriguing bridging fashions. ${ }^{19}$ It is noted that investigation of the substituent position isomerization influence of oxidazole-pyridine ligands on the formation of coordination polymers is still scarcely reported. Especially, to the best of our knowledge, 3-(5-methyl-1,3,4-oxadiazol-2-yl)pyridine $\left(\mathrm{L}_{1}\right)$ and 4(5-methyl-1,3,4-oxadiazol-2-yl)pyridine $\left(\mathrm{L}_{2}\right)$ are never reported in the coordination chemistry. The development of new ligand systems is continuously an important aspect for the chemistry of metal-organic coordination polymer. Previously we also reported a series of functional coordination polymers using versatile N-containing ligands. ${ }^{20}$ In this work two positionisomeric oxadiazol-pyridine ligands $\mathrm{L}_{1}$ and $\mathrm{L}_{2}$ have been employed, a series of novel coordination polymers, namely $\left[\mathrm{Cd}_{2}\left(\mu_{2}-\mathrm{L}_{1}\right)_{2}\left(\mu_{2}-\mathrm{NCS}\right)_{4}\right]_{n}(\mathbf{1}),\left\{\left[\mathrm{Cd}\left(\mathrm{L}_{1}\right)\left(\mu_{2}-\mathrm{dca}\right)_{2}\left(\mathrm{H}_{2} \mathrm{O}\right)\right] \cdot \mathrm{H}_{2} \mathrm{O}\right\}_{n}$ (2), $\left\{\left[\mathrm{Cu}\left(\mu_{2}-\mathrm{L}_{1}\right)_{2}(\mathrm{NCS})_{2}\right] \cdot 0.5 \mathrm{H}_{2} \mathrm{O}\right\}_{n} \quad(3), \quad\left\{\left[\mathrm{Ag}_{2}\left(\mu_{2}-\mathrm{L}_{1}\right)\left(\mu_{3}-\mathrm{L}_{1}\right)_{2}\right] \cdot 2 \mathrm{PF}_{6}\right\}_{n}$ (4), $\left\{\left[\mathrm{Ag}_{3}\left(\mu_{2}-\mathrm{L}_{1}\right)_{4}\left(\mu_{2}-\mathrm{CF}_{3} \mathrm{SO}_{3}\right)\left(\mathrm{CF}_{3} \mathrm{SO}_{3}\right)\right] \cdot \mathrm{CF}_{3} \mathrm{SO}_{3}\right\} \quad(5), \quad\left\{\left[\mathrm{Cd}\left(\mathrm{L}_{2}\right)_{2}\right.\right.$ $\left.\left.\left(\mu_{2}-\mathrm{NCS}\right)_{2}\right]\right\}_{n}$ (6), $\left[\mathrm{Ag}\left(\mu_{2}-\mathrm{L}_{2}\right)\left(\mu_{2}-\mathrm{CF}_{3} \mathrm{SO}_{3}\right)\right]_{n}$ (7) and $\left\{\left[\mathrm{Ag}\left(\mu_{2}-\mathrm{L}_{2}\right)\right]\right.$. $\left.\mathrm{BF}_{4}\right\}_{n}$ (8) have been isolated. Variable temperature magnetic susceptibility measurements $(2-300 \quad \mathrm{~K})$ reveal antiferromagnetic interactions between central copper(II) ions for 3. Solid-state luminescent properties of 1, 2 and 4-8 have been investigated indicating strong fluorescent emissions. Additionally, luminescent measurements illustrate that 8 exhibits highly sensitive luminescence sensing for $\mathrm{Cr}_{2} \mathrm{O}_{7}{ }^{2-}$ in aqueous solutions with high quenching efficiency $K_{\mathrm{sv}}=2.08 \times 10^{4} \mathrm{~L}$ $\mathrm{mol}^{-1}$ and low detection limit $(0.19 \mu \mathrm{M}(\mathrm{S} / \mathrm{N}=3))$, which make it a promising candidate for sensing $\mathrm{Cr}_{2} \mathrm{O}_{7}{ }^{2-}$ ions in practical. Luminescent measurements illustrate that 8 also represents the first report of coordination polymers based on oxadiazol-pyridine derivatives as luminescent response to $\mathrm{Cr}_{2} \mathrm{O}_{7}{ }^{2-}$ ions in the water solutions. Different coordination modes of $\mathrm{L}_{1}$ and $\mathrm{L}_{2}$ are also briefly discussed, which indicates these isomeric oxadiazolpyridine building blocks have great potential in the construction of these unique metal organic coordination polymers.

\section{Experimental section}

\section{General methods}

Ligands $\mathrm{L}_{1}$ and $\mathrm{L}_{2}$ were prepared by literature methods. ${ }^{21}$ All the other reagents were purchased commercially and used without further purification. Deionized water was used as solvent in this work. C, H, and N microanalyses were carried out with a PerkinElmer 240 elemental analyzer. FT-IR spectra were recorded from $\mathrm{KBr}$ pellets in the range $4000-400 \mathrm{~cm}^{-1}$ on a Bio-Rad FTS 135 spectrometer. Variable-temperature magnetic susceptibilities were measured using a MPMS-7 SQUID magnetometer. Diamagnetic corrections were made with Pascal's constants for all constituent atoms. Photoluminescence spectra were measured by MPF-4 fluorescence spectrophotometer with a xenon arc lamp as the light source.

Synthesis of 3-(5-methyl-1,3,4-oxadiazol-2-yl)pyridine $\left(L_{1}\right)$. $35 \mathrm{mmol}$ of the nicotinohydrazide were suspended in $95 \mathrm{mmol}$ (2.7 eq.) triethyl orthoacetate. The reaction mixture was heated under reflux for 12 hours. After cooling to ambient temperature the formed solid was filtered off, washed twice with $15 \mathrm{~mL}$ of diethylether and dried in air. Yield: $70 \%$ of theory. ${ }^{1} \mathrm{H}-\mathrm{NMR}(400$ MHz, DMSO-d $\left.{ }_{6}\right): \delta=2.61 \mathrm{ppm}\left(\mathrm{s}, 3 \mathrm{H}, \mathrm{CH}_{3}\right) ; 7.88-7.90(\mathrm{~m}, 2 \mathrm{H}$, arom. C-H); 8.80-8.82 (m, 2H, arom. C-H). ${ }^{13} \mathrm{C}-\mathrm{NMR}(100 \mathrm{MHz}$, DMSO-d $\left.{ }_{6}\right): \delta=10.7$ ppm (s, 1C, $\left.\mathrm{CH}_{3}\right) ; 119.9$ (s, 2C, arom. C-H); $130.5\left(\mathrm{~s}, 1 \mathrm{C}, \mathrm{C}_{\mathrm{q}}\right) ; 150.9$ (s, 2C, arom. $\left.\mathrm{C}-\mathrm{H}\right) ; 162.4\left(\mathrm{~s}, 1 \mathrm{C}, \mathrm{C}_{\mathrm{q}}=\mathrm{N}\right)$; $165.0\left(\mathrm{~s}, 1 \mathrm{C}, \mathrm{C}_{\mathrm{q}}=\mathrm{N}\right)$. FT-IR $\left(\mathrm{cm}^{-1}, \mathrm{KBr}\right): 1565(\mathrm{~m}), 1549(\mathrm{w})$, 1465(m), 1430(m), 1349(w), 1247(w), 1131(m), 1086(m), 1044(m), 1014(m), 823(m), 705(m).

Synthesis of 4-(5-methyl-1,3,4-oxadiazol-2-yl)pyridine $\left(\mathrm{L}_{2}\right)$. $35 \mathrm{mmol}$ of the 4-pyridinecarboxylic hydrazide were suspended in $95 \mathrm{mmol}$ (2.7 eq.) triethyl orthoacetate. The reaction mixture was heated under reflux for 12 hours. After cooling to ambient temperature the formed solid was filtered off, washed twice with $15 \mathrm{~mL}$ of diethylether and dried in air. Yield: $55 \%$ of theory. ${ }^{1} \mathrm{H}$ NMR (400 MHz, DMSO-d $\left.{ }_{6}\right): \delta=2.60 \mathrm{ppm}\left(\mathrm{s}, 3 \mathrm{H}, \mathrm{CH}_{3}\right) ; 7.61-7.64$ (m, $1 \mathrm{H}$, arom. C-H); 8.32-8.34 (m, 1H, arom. C-H); 8.78-8.79 (m, 1H, arom. C-H); 9.13 (s, 1H, arom. $\mathrm{N}=\mathrm{C}-\mathrm{H}) .{ }^{13} \mathrm{C}\left\{{ }^{1} \mathrm{H}\right\}-\mathrm{NMR}$ $\left(100 \mathrm{MHz}, \mathrm{DMSO}-\mathrm{d}_{6}\right): \delta=10.6 \mathrm{ppm}\left(\mathrm{s}, 1 \mathrm{C}, \mathrm{CH}_{3}\right) ; 120.0\left(\mathrm{~s}, 1 \mathrm{C}, \mathrm{C}_{\mathrm{q}}\right)$; 124.3 (s, 1C, $\mathrm{C}_{\mathrm{q}}$ ); 133.9 (s, 1C, arom. C-H); 146.9 (s, 1C, arom. C$\mathrm{H}) ; 152.2$ (s, 1C, arom. C-H); 164.4 (s, 1C, arom. C-H); 162.1 (s, $\left.1 \mathrm{C}, \mathrm{C}_{\mathrm{q}}=\mathrm{N}\right)$. FT-IR $\left(\mathrm{cm}^{-1}, \mathrm{KBr}\right): 1585(\mathrm{~m}), 1538(\mathrm{w}), 1413(\mathrm{~m})$, 1430(m), 1354(w), 1271(m), 1142(m), 1033(m), 834(m), 770(m).

\section{Preparation of compounds 1-8}

$\left[\mathrm{Cd}_{2}\left(\mu_{2}-\mathbf{L}_{1}\right)_{2}\left(\mu_{2}-\mathbf{N C S}\right)_{4}\right]_{n}$ (1). A mixture of $\mathrm{CdCl}_{2}$ (18.33 mg, $0.10 \mathrm{mmol}), \mathrm{L}_{1}(17.71 \mathrm{mg}, 0.10 \mathrm{mmol}), \mathrm{NH}_{4} \mathrm{NCS}(15.23 \mathrm{mg}, 0.20$ $\mathrm{mmol})$ and water $(20 \mathrm{~mL})$ was refluxed and stirred for $12 \mathrm{~h}$, then the resulting solutions were filtered. The filtrate was kept in a $\mathrm{CaCl}_{2}$ desiccator. After a few days suitable colorless single crystals were obtained for X-ray diffraction study, yield $55 \%$. Elemental analysis calcd (\%) for $\mathrm{C}_{20} \mathrm{H}_{14} \mathrm{Cd}_{2} \mathrm{~N}_{10} \mathrm{O}_{2} \mathrm{~S}_{4}$ : C $30.82 \%$, H 1.81\%, N 17.97\%; found: C 30.97\%, H 1.93\%, N 18.16\%. FT-IR $\left(\mathrm{cm}^{-1}, \mathrm{KBr}\right):$ 2090(s), 1572(m), 1558(w), 1465(m), 1263(w), 1131(m), 1098(m), 1033(m), 1014(m), 814(m), 719(m).

$\left\{\left[\mathbf{C d}\left(\mu_{2}-\mathbf{L}_{1}\right)\left(\mu_{2}-d c a\right)_{2}\left(\mathbf{H}_{2} \mathbf{O}\right)\right] \cdot \mathbf{H}_{2} \mathbf{O}\right\}_{n}$ (2). A mixture of $\mathrm{CdCl}_{2}$ (18.33 mg, $0.10 \mathrm{mmol}), \mathrm{L}_{1}(17.71 \mathrm{mg}, 0.10 \mathrm{mmol})$, Nadca (17.81 $\mathrm{mg}, 0.20 \mathrm{mmol})$ and water $(20 \mathrm{~mL})$ was refluxed and stirred for $12 \mathrm{~h}$, then the resulting solutions were filtered. The filtrate was kept in a $\mathrm{CaCl}_{2}$ desiccator. After a few days suitable colorless single crystals were obtained for X-ray diffraction study, yield 57\%. Elemental analysis calcd (\%) for $\mathrm{C}_{21} \mathrm{H}_{11} \mathrm{CdN}_{9} \mathrm{O}_{3}$ : C 45.88\%, $\mathrm{H} 2.02 \%$, N 22.93\%; found: $\mathrm{C}$ $46.15 \%, \mathrm{H} 2.16 \%, \mathrm{~N} 23.12 \%$. FT-IR $\left(\mathrm{cm}^{-1}, \mathrm{KBr}\right): 3500(\mathrm{~m})$, 2170(s), 1570(m), 1550(w), 1352(m), 1260(w), 1129(m), 1080(m), 1035(m), 1015(m), 815(m), 720(m). 
$\left\{\left[\mathbf{C u}\left(\mu_{2}-\mathbf{L}_{1}\right)_{2}(\mathbf{N C S})_{2}\right] \cdot 0.5 \mathbf{H}_{2} \mathbf{O}\right\}_{n}$ (3). A mixture of $\mathrm{CuCl}_{2}$ (17.05 mg, $0.10 \mathrm{mmol}), \mathrm{L}_{1}(17.71 \mathrm{mg}, 0.10 \mathrm{mmol}), \mathrm{NH}_{4} \mathrm{NCS}$ $(15.23 \mathrm{mg}, 0.20 \mathrm{mmol})$ and water $(20 \mathrm{~mL})$ was refluxed and stirred for $12 \mathrm{~h}$, then the resulting solutions were filtered. The filtrate was kept in a $\mathrm{CaCl}_{2}$ desiccator. After a few days suitable green single crystals were obtained for X-ray diffraction study, yield $37 \%$. Elemental analysis calcd (\%) for $\mathrm{C}_{18} \mathrm{H}_{14.5} \mathrm{CuN}_{8} \mathrm{O}_{2.5} \mathrm{~S}_{2}$ : C $42.35 \%, \mathrm{H} 2.86 \%, \mathrm{~N} 21.95 \%$; found: C $42.46 \%, \mathrm{H} 2.97 \%, \mathrm{~N}$ $22.13 \%$. FT-IR ( $\left.\mathrm{cm}^{-1}, \mathrm{KBr}\right): 3410(\mathrm{~m}), 2090(\mathrm{~s}), 1557(\mathrm{~m}), 1420(\mathrm{~m})$, 1359(w), 1268(m), 1093(m), 816(m), 719(m).

$\left\{\left[\mathbf{A g}_{2}\left(\mu_{2}-\mathbf{L}_{1}\right)_{3}\right] \cdot 2 \mathbf{P F}_{6}\right\}_{n}(4)$. A mixture of $\operatorname{AgPF}_{6}(25.29 \mathrm{mg}, 0.10$ $\mathrm{mmol}), \mathrm{L}_{1}(17.71 \mathrm{mg}, 0.10 \mathrm{mmol})$ and water $(20 \mathrm{~mL})$ was refluxed and stirred for $12 \mathrm{~h}$, then the resulting solutions were filtered. The filtrate was kept in a $\mathrm{CaCl}_{2}$ desiccator. After a few days colorless suitable single crystals were obtained for X-ray diffraction study, yield 59\%. Elemental analysis calcd (\%) for $\mathrm{C}_{24} \mathrm{H}_{21} \mathrm{Ag}_{2} \mathrm{~F}_{12} \mathrm{~N}_{9} \mathrm{O}_{3} \mathrm{P}_{2}$ : C $29.14 \%, \mathrm{H} 2.14 \%$, N 12.74\%; found: $\mathrm{C}$ $29.34 \%, \mathrm{H} 2.38 \%$, N $12.97 \%$. FT-IR $\left(\mathrm{cm}^{-1}, \mathrm{KBr}\right): 1589(\mathrm{~m})$, $1420(\mathrm{~m}), 1270(\mathrm{~m}), 1110(\mathrm{~m}), 838(\mathrm{~s}), 704(\mathrm{~m})$.

$\left\{\left[\mathbf{A g}_{3}\left(\mu_{2}-\mathbf{L}_{1}\right)_{4}\left(\mu_{2}-\mathbf{C F}_{3} \mathbf{S O}_{3}\right)\left(\mathbf{C F}_{3} \mathbf{S O}_{3}\right)\right] \cdot \mathbf{C F}_{3} \mathbf{S O}_{3}\right\}_{n}(\mathbf{5})$. A mixture of $\mathrm{AgCF}_{3} \mathrm{SO}_{3}(25.69 \mathrm{mg}, 0.10 \mathrm{mmol}), \mathrm{L}_{1}(17.71 \mathrm{mg}, 0.10 \mathrm{mmol})$ and water $(20 \mathrm{~mL})$ was refluxed and stirred for $12 \mathrm{~h}$, then the resulting solutions were filtered. The filtrate was kept in a $\mathrm{CaCl}_{2}$ desiccator. After a few days suitable single crystals were obtained for X-ray diffraction study, yield 39\%. Elemental analysis calcd (\%) for $\mathrm{C}_{35} \mathrm{H}_{28} \mathrm{Ag}_{3} \mathrm{~F}_{9} \mathrm{~N}_{12} \mathrm{O}_{13} \mathrm{~S}_{3}$ : C $29.70 \%, \mathrm{H} 1.99 \%$, N $11.87 \%$; found: $\mathrm{C}$ 29.96\%, H 2.06\%, N 11.96\%. FT-IR ( $\left.\mathrm{cm}^{-1}, \mathrm{KBr}\right): 1589(\mathrm{~m})$, 1410(m), 1275(m), 1168(m), 820(m), 1031(bm), 827(m), 720(m).

$\left\{\left[\mathbf{C d}\left(\mathbf{L}_{2}\right)_{2}\left(\mu_{2}-\mathrm{NCS}\right)_{2}\right]\right\}_{n}(\mathbf{6}) .6$ was prepared through the similar synthetic method of 3 only using $\mathrm{L}_{2}(17.71 \mathrm{mg}, 0.10 \mathrm{mmol})$ to replace $\mathrm{L}_{1}(17.71 \mathrm{mg}, 0.10 \mathrm{mmol})$ in the reaction system. After a few days suitable colorless single crystals were obtained for $\mathrm{X}$ ray diffraction study, yield $41 \%$. Elemental analysis calcd (\%) for $\mathrm{C}_{18} \mathrm{H}_{14} \mathrm{CdN}_{8} \mathrm{O}_{2} \mathrm{~S}_{2}$ : C $39.24 \%, \mathrm{H} 2.56 \%, \mathrm{~N} 20.34 \%$; found: $\mathrm{C}$ $39.57 \%, \mathrm{H} 2.79 \%$, N 20.59\%. FT-IR $\left(\mathrm{cm}^{-1}, \mathrm{KBr}\right): 2100$ (s), $1617(\mathrm{~m}), 1567(\mathrm{~m}), 1251(\mathrm{~m}), 728(\mathrm{~m})$.

$\left[\mathbf{A g}\left(\mu_{2}-\mathbf{L}_{2}\right) \cdot\left(\mu_{2}-\mathbf{C F}_{3} \mathbf{S O}_{3}\right)\right]_{n}(7) .7$ was prepared through the similar synthetic method of 5 only using $\mathrm{L}_{2}(17.71 \mathrm{mg}, 0.10$ mmol) to replace $\mathrm{L}_{1}(17.71 \mathrm{mg}, 0.10 \mathrm{mmol})$ in the reaction system. After a few days suitable colorless single crystals were obtained for X-ray diffraction study, yield $36 \%$. Elemental analysis calcd (\%) for $\mathrm{C}_{9} \mathrm{H}_{7} \mathrm{Ag}_{2} \mathrm{~F}_{3} \mathrm{~N}_{3} \mathrm{O}_{4} \mathrm{~S}: \mathrm{C} 20.55 \%, \mathrm{H} 1.34 \%$, N 7.99\%; found: C $20.76 \%$, H $1.57 \%$, N 8.16\%. FT-IR ( $\left.\mathrm{cm}^{-1}, \mathrm{KBr}\right): 1616(\mathrm{~m})$, 1565(m), 1418(m), 1272(m), 1142(m), 1033(m), 834(m), 707(m).

$\left\{\left[\mathbf{A g}\left(\mu_{2}-\mathbf{L}_{2}\right)\right] \cdot \mathbf{B F}_{4}\right\}_{n}(8)$. A mixture of $\mathrm{AgBF}_{4}(0.10 \mathrm{mmol}), \mathrm{L}_{2}$ $(0.10 \mathrm{mmol})$ and water $(20 \mathrm{~mL})$ was refluxed and stirred for $12 \mathrm{~h}$, then the resulting solutions were filtered. The filtrate was kept in a $\mathrm{CaCl}_{2}$ desiccator. After a few days suitable single crystals were obtained for X-ray diffraction study, yield $46 \%$. Elemental analysis calcd (\%) for $\mathrm{C}_{16} \mathrm{H}_{14} \mathrm{AgBF}_{4} \mathrm{~N}_{6} \mathrm{O}_{2}$ : C $37.17 \%, \mathrm{H} 2.73 \%, \mathrm{~N}$ 16.26\%; found: C $37.34 \%$, H 2.97\%, N $16.46 \%$. FT-IR ( $\left.\mathrm{cm}^{-1}, \mathrm{KBr}\right):$ 1616(m), 1566(m), 1416(m), 1266(m), 1051(bm), 836(m), 725(m).

\section{X-ray crystallography}

Diffraction intensities for complexes 1-8 were collected on a Bruker SMART 1000 CCD diffractometer with graphite- monochromated Mo-K $\alpha$ radiation $(\lambda=0.71073 \AA)$ by using the $\omega-\varphi$ scan technique. Lorentz polarization and MULTISCAN absorption corrections were applied. The structures were solved by direct methods and refined with the full-matrix least-squares technique using the SHELXS-97 and SHELXL-97 programs. ${ }^{22,23}$ Anisotropic thermal parameters were assigned to all non-hydrogen atoms. The positions of hydrogen atoms of the water molecules were located from difference maps and refined with isotropic temperature factors. The positions of other hydrogen atoms were generated geometrically. Analytical expressions of neutral-atom scattering factors were employed, and anomalous dispersion corrections were incorporated. The crystallographic data and details of refinements for compounds 1-8 are summarized in Table 1. Selected bond lengths and angles are listed in Table 2. Corresponding hydrogen bonds lengths and angles for 1-8 are listed in Table S1. $\dagger$

\section{Results and discussion}

The crystal samples of complexes 1-8 can retain their crystalline integrity at room temperature for two weeks. As shown in Schemes 1 and 2, only nitrogen atoms of oxadiazole and pyridine rings can participate in coordination and the steric effect prevents oxadiazole oxygen atoms from metal coordination. There are five different coordination modes in 1-8: for 1, 3 and 5 , these $\mathrm{L}_{1}$ ligands in these coordination polymers contain bridging coordination modes a. For 2 , these $\mathrm{L}_{1}$ ligands contain mono-dentate coordination mode b, while in $\mathbf{4}$ two different coordination (bidentate bridging mode $\mathrm{a}$ and tridenate bridging mode c) for $\mathrm{L}_{1}$ can be observed. For $\mathbf{6}$, mono-dentate coordination mode $\mathrm{d}$ for $\mathrm{L}_{2}$ can be observed while for 7-8 bidentate bridging coordination mode for $\mathrm{L}_{2}$ can be observed. These various different coordination modes also draw the selfassembly of coordination polymers to form different coordination polymers.

\section{Structure of $\left[\mathrm{Cd}_{2}\left(\mu_{2}-\mathrm{L}_{1}\right)_{2}\left(\mu_{2}-\mathrm{NCS}\right)_{4}\right]_{n}(\mathbf{1})$}

As shown in Fig. 1, the asymmetric structural unit of $\left[\mathrm{Cd}_{2}\left(\mu_{2}-\right.\right.$ $\left.\left.\mathrm{L}_{1}\right)_{2}\left(\mu_{2}-\mathrm{NCS}\right)_{4}\right]_{n}(\mathbf{1})$ contains two central Cd ${ }^{\mathrm{II}}$ ions (Cd1 and $\left.\mathrm{Cd} 2\right)$, two $\mu_{2}$-bridging $\mathrm{L}_{1}$ ligands and two $\mu_{2}$-bridging $\mathrm{SCN}^{-}$groups. The central $\mathrm{Cd}^{\mathrm{II}}$ atom is six-coordinated by two nitrogen atoms from $\mathrm{L}_{1}$, two $\mathrm{SCN}^{-}$sulfur atoms and two $\mathrm{SCN}^{-}$nitrogen atoms forming $\mathrm{CdN}_{4} \mathrm{~S}_{2}$ donor set. The $\mathrm{Cd}-\mathrm{N}$ distances vary from 2.302(4) to 2.431(2) $\AA$, Cd-S distances are 2.7140(15) $\AA$, all the N$\mathrm{Cd}-\mathrm{N}$ and $\mathrm{N}-\mathrm{Cd}-\mathrm{S}$ angles fall in the range of 83.24(12)94.27(9) ${ }^{\circ}$, such coordination mode is in line with an octahedrally coordinated $\mathrm{Cd}^{\mathrm{II}}$ center.

As shown in Fig. 2, these thiocyanate ligands adopt bidentate bridging coordination mode, which link these neighboring $\mathrm{Cd}^{\mathrm{II}}$ centers forming $\{\mathrm{Cd}-\mathrm{NCS}-\mathrm{Cd}\}$ chains. The shortest $\mathrm{Cd} \cdots \mathrm{Cd}$ distance within the $\{\mathrm{Cd}-\mathrm{NCS}-\mathrm{Cd}\}$ chains is 5.714(20) $\AA$. On the other hand, as listed in Scheme 2(a), these $\mathrm{L}_{1}$ ligands in $\mathbf{1}$ adopt $\mu_{2}$-bridging coordination mode. These $\{\mathrm{Cd}-\mathrm{NCS}-\mathrm{Cd}\}$ chains are further interlinked via bi-dentate $\mathrm{L}_{1}$ ligands forming the $2 \mathrm{D}$ coordination polymer. These $\mathrm{L}_{1}$ carbon atoms $(\mathrm{C}(4)$ and $\mathrm{C}(8))$ 


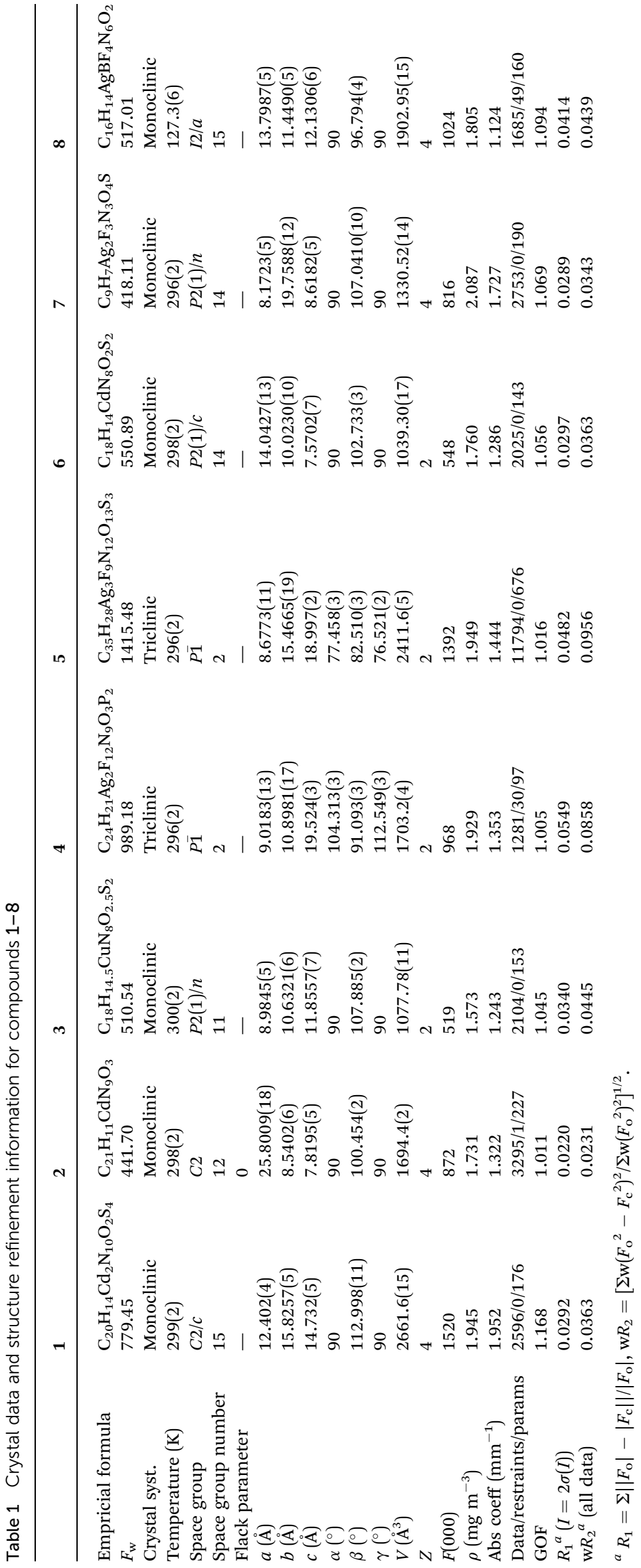


Table 2 Selected bond lengths $(\AA)$ and angles $\left({ }^{\circ}\right)$ for $1-8^{a}$

1

$\mathrm{Cd}(1)-\mathrm{N}(4 \mathrm{~A})$

$\mathrm{Cd}(1)-\mathrm{N}(3 \mathrm{~A})$

$\mathrm{Cd}(1)-\mathrm{S}(2 \mathrm{~A})$

$\mathrm{Cd}(2)-\mathrm{N}(5)$

$\mathrm{N}(4 \mathrm{~A})-\mathrm{Cd}(1)-\mathrm{N}(4)$

$\mathrm{N}(4)-\mathrm{Cd}(1)-\mathrm{N}(3 \mathrm{~A})$

$\mathrm{N}(4)-\mathrm{Cd}(1)-\mathrm{N}(3)$

2

$\mathrm{Cd}(1)-\mathrm{N}(4)$

$\mathrm{Cd}(1)-\mathrm{N}(7)$

$\mathrm{N}(4)-\mathrm{Cd}(1)-\mathrm{N}(6 \mathrm{~A})$

$\mathrm{N}(6 \mathrm{~A})-\mathrm{Cd}(1)-\mathrm{N}(7)$

$\mathrm{N}(6 \mathrm{~A})-\mathrm{Cd}(1)-\mathrm{N}(9 \mathrm{~A})$

$\mathrm{N}(6)-\mathrm{Cd}(1)-\mathrm{N}(1)$

$\mathrm{N}(9 \mathrm{~A})-\mathrm{Cd}(1)-\mathrm{N}(1)$

3

$\mathrm{Cu}(1)-\mathrm{N}(4)$

$\mathrm{Cu}(1)-\mathrm{N}(1)$

$\mathrm{N}(4)-\mathrm{Cu}(1)-\mathrm{N}(4 \mathrm{~A})$

$\mathrm{N}(4 \mathrm{~A})-\mathrm{Cu}(1)-\mathrm{N}(1)$

4

$\mathrm{Ag}(1)-\mathrm{N}(2)$

$\mathrm{Ag}(1)-\mathrm{N}(8)$

$\mathrm{Ag}(2)-\mathrm{N}(6)$

$\mathrm{N}(2)-\mathrm{Ag}(1)-\mathrm{N}(4 \mathrm{~A})$

$\mathrm{N}(4 \mathrm{~A})-\mathrm{Ag}(1)-\mathrm{N}(8)$

5

$\mathrm{Ag}(1)-\mathrm{N}(4)$

$\mathrm{Ag}(1)-\mathrm{N}(1)$

$\mathrm{Ag}(2)-\mathrm{N}(3)$

$\mathrm{N}(4)-\mathrm{Ag}(1)-\mathrm{N}(1)$

$\mathrm{N}(7)-\mathrm{Ag}(2)-\mathrm{N}(3)$

$\mathrm{N}(3)-\mathrm{Ag}(2)-\mathrm{N}(6)$

6

$\mathrm{Cd}(1)-\mathrm{N}(4)$

$\mathrm{Cd}(1)-\mathrm{N}(1)$

$\mathrm{N}(4)-\mathrm{Cd}(1)-\mathrm{N}(4 \mathrm{~A})$

$\mathrm{N}(4 \mathrm{~A}) \# 1-\mathrm{Cd}(1)-\mathrm{N}(1)$

7

$\operatorname{Ag}(1)-\mathrm{N}(1)$

$\mathrm{Ag}(1)-\mathrm{O}(4)$

$\mathrm{N}(1)-\mathrm{Ag}(1)-\mathrm{O}(2 \mathrm{~A})$

8

$\mathrm{Ag}(1)-\mathrm{N}(1)$

$\mathrm{Ag}(1)-\mathrm{N}(3 \mathrm{~B})$

$\mathrm{N}(1)-\mathrm{Ag}(1)-\mathrm{N}(1)$

$\mathrm{N}(1)-\mathrm{Ag}(1)-\mathrm{N}(3)$

$2.302(4)$
$2.431(3)$
$2.7140(15)$
$2.291(4)$
$102.64(18)$
$83.24(12)$
$170.09(13)$

2.261(3)

$2.326(4)$

98.40(13)

91.66(17)

93.34(14)

93.57(14)

$89.37(13)$

$1.974(2)$
$2.0578(19)$
180.0
$91.01(8)$

2.277(4)

2.309(4)

2.225(4)

124.79(16)

109.87(16)

2.209(4)

2.458(4)

2.297(4)

103.68(13)

143.19(14)

104.96(13)

$2.325(3)$
$2.364(2)$
$180.00(15)$
$89.49(9)$

2.165(2)

2.710(2)

99.27(1)

$2.282(3)$
$2.349(3)$
$121.29(18)$
$94.85(12)$
$\mathrm{Cd}(1)-\mathrm{N}(4)$

$\mathrm{Cd}(1)-\mathrm{N}(3)$

$\mathrm{Cd}(1)-\mathrm{S}(2)$

$\mathrm{N}(4)-\mathrm{Cd}(1)-\mathrm{S}(2 \mathrm{~A})$

$\mathrm{N}(4 \mathrm{~A})-\mathrm{Cd}(1)-\mathrm{N}(3 \mathrm{~A})$

$\mathrm{N}(4 \mathrm{~A})-\mathrm{Cd}(1)-\mathrm{N}(3)$

$\mathrm{N}(3 \mathrm{~A})-\mathrm{Cd}(1)-\mathrm{N}(3)$

$\mathrm{Cd}(1)-\mathrm{N}(6 \mathrm{~A})$

Cd(1)-N(9A)

$\mathrm{N}(4)-\mathrm{Cd}(1)-\mathrm{N}(7)$

$\mathrm{N}(4)-\mathrm{Cd}(1)-\mathrm{N}(9 \mathrm{~A})$

$\mathrm{N}(7)-\mathrm{Cd}(1)-\mathrm{N}(9 \mathrm{~A})$

$\mathrm{N}(7)-\mathrm{Cd}(1)-\mathrm{O}(2)$

$\mathrm{N}(1)-\mathrm{Cd}(1)-\mathrm{O}(2)$

$\mathrm{Cu}(1)-\mathrm{N}(4 \mathrm{~A})$

$\mathrm{N}(4 \mathrm{~A})-\mathrm{Cu}(1)-\mathrm{N}(1 \mathrm{~A})$

$\mathrm{N}(4)-\mathrm{Cu}(1)-\mathrm{N}(1)$

$\mathrm{N}(4)-\mathrm{Cu}(1)-\mathrm{N}(1 \mathrm{~A})$

$\mathrm{Ag}(1)-\mathrm{N}(4 \mathrm{~A})$

$\mathrm{Ag}(1)-\mathrm{N}(3)$

$\mathrm{Ag}(2)-\mathrm{N}(9 \mathrm{~A})$

$\mathrm{N}(2)-\mathrm{Ag}(1)-\mathrm{N}(8)$

$\mathrm{N}(2)-\mathrm{Ag}(1)-\mathrm{N}(3)$

$\mathrm{Ag}(1)-\mathrm{N}(12 \mathrm{~A})$

$\mathrm{Ag}(2)-\mathrm{N}(7)$

$\mathrm{Ag}(2)-\mathrm{N}(6)$

$\mathrm{N}(12 \mathrm{~A})-\mathrm{Ag}(1)-\mathrm{N}(1)$

$\mathrm{N}(7)-\mathrm{Ag}(2)-\mathrm{N}(6)$

$\mathrm{N}(7)-\mathrm{Ag}(2)-\mathrm{O}(5)$

$\mathrm{Cd}(1)-\mathrm{N}(4 \mathrm{~A})$

$\mathrm{Cd}(1)-\mathrm{N}(1 \mathrm{~A})$

$\mathrm{N}(4)-\mathrm{Cd}(1)-\mathrm{N}(1)$

$\mathrm{N}(1)-\mathrm{Cd}(1)-\mathrm{S}(1 \mathrm{~B})$

$\mathrm{Ag}(1)-\mathrm{N}(3 \mathrm{~A})$

$\mathrm{N}(1)-\mathrm{Ag}(1)-\mathrm{N}(3 \mathrm{~A})$

$\mathrm{N}(3 \mathrm{~A})-\mathrm{Ag}(1)-\mathrm{O}(4)$

$\mathrm{Ag}(1)-\mathrm{N}(1)$

$\mathrm{N}(1)-\mathrm{Ag}(1)-\mathrm{N}(3 \mathrm{~A})$

$\mathrm{N}(1)-\mathrm{Ag}(1)-\mathrm{N}(3)$
$2.302(4)$
$2.431(3)$
$2.7140(15)$
$93.70(10)$
$170.09(13)$
$83.24(12)$
$92.13(16)$

2.264(4)

2.326(3)

92.38(14)

168.22(14)

$86.39(16)$

$86.59(13)$

88.01(9)

1.974(2)

$88.99(8)$

$88.99(8)$

91.01(8)

2.307(4)

$2.406(4)$

2.231(4)

111.05(14)

102.49(14)

2.211(4)

2.284(3)

2.352(4)

104.13(14)

109.20(13)

94.06(14)

$2.325(3)$
$2.364(2)$
$90.51(9)$
$90.37(6)$

2.184(2)

164.72(9)

83.54(1)

$2.282(3)$
$125.45(12)$
$125.45(12)$
$\mathrm{Cd}(2)-\mathrm{N}(1)$

$\mathrm{Cd}(2)-\mathrm{N}(1 \mathrm{~A})$

$\mathrm{Cd}(2)-\mathrm{N}(5 \mathrm{~B})$

$\mathrm{N}(3 \mathrm{~A})-\mathrm{Cd}(1)-\mathrm{S}(2 \mathrm{~A})$

$\mathrm{N}(4 \mathrm{~A})-\mathrm{Cd}(1)-\mathrm{S}(2)$

$\mathrm{N}(3 \mathrm{~A})-\mathrm{Cd}(1)-\mathrm{S}(2)$

$\mathrm{S}(2 \mathrm{~A})-\mathrm{Cd}(1)-\mathrm{S}(2)$

$\mathrm{Cd}(1)-\mathrm{N}(1)$

$\mathrm{Cd}(1)-\mathrm{O}(2)$

$\mathrm{N}(4)-\mathrm{Cd}(1)-\mathrm{N}(1)$

$\mathrm{N}(7)-\mathrm{Cd}(1)-\mathrm{N}(1)$

$\mathrm{N}(4)-\mathrm{Cd}(1)-\mathrm{O}(2)$

$\mathrm{N}(6 \mathrm{~A})-\mathrm{Cd}(1)-\mathrm{O}(2)$

$\mathrm{N}(9 \mathrm{~A})-\mathrm{Cd}(1)-\mathrm{O}(2)$

$\mathrm{Cu}(1)-\mathrm{N}(1 \mathrm{~A})$

$\mathrm{N}(1)-\mathrm{Cu}(1)-\mathrm{N}(1 \mathrm{~A})$

$\mathrm{N}(4)-\mathrm{Cu}(1)-\mathrm{N}(1 \mathrm{~A})$

$\mathrm{Ag}(2)-\mathrm{N}(7)$

$\mathrm{Ag}(2)-\mathrm{N}(1)$

$\mathrm{N}(4 \mathrm{~A})-\mathrm{Ag}(1)-\mathrm{N}(3)$

$\mathrm{N}(6)-\mathrm{Ag}(2)-\mathrm{N}(9 \mathrm{~A})$

$\mathrm{N}(8)-\mathrm{Ag}(1)-\mathrm{N}(3)$

$\mathrm{Ag}(3)-\mathrm{N}(10)$

$\mathrm{Ag}(3)-\mathrm{N}(9)$

$\mathrm{Ag}(2)-\mathrm{O}(5)$

$\mathrm{N}(3)-\mathrm{Ag}(2)-\mathrm{O}(5)$

$\mathrm{N}(9)-\mathrm{Ag}(3)-\mathrm{N}(10)$

$\mathrm{N}(6)-\mathrm{Ag}(2)-\mathrm{O}(5)$

2.421(4)

$2.554(4)$

98.30(17)

152.34(16)

108.07(14)

2.154(3)

$2.152(3)$

2.591(4)

$77.79(13)$

175.45(15)

123.73(15)

$\mathrm{Cd}(1)-\mathrm{S}(1 \mathrm{~A})$

Cd(1)-S(1B)

$\mathrm{N}(4)-\mathrm{Cd}(1)-\mathrm{S}(1 \mathrm{~A})$

$\mathrm{N}(1)-\mathrm{Cd}(1)-\mathrm{S}(1 \mathrm{~A})$

2.7253(9)

$2.7253(9)$

91.75(8)

89.63(6)

$\mathrm{Ag}(1)-\mathrm{O}(2 \mathrm{~A})$

2.623(2)

$\mathrm{N}(1)-\mathrm{Ag}(1)-\mathrm{O}(4)$

94.19(1)

$\mathrm{Ag}(1)-\mathrm{N}(3 \mathrm{~A}) \# 1$
$\mathrm{~N}(3 \mathrm{~A})-\mathrm{Ag}(1)-\mathrm{N}$

$\mathrm{N}(1)-\mathrm{Ag}(1)-\mathrm{N}(3)$
$2.349(3)$

94.65(17)

$94.85(12)$

${ }^{a}$ Symmetry transformations used to generate equivalent atoms: for 1 : (A) $-x, y,-z+1 / 2 ;$ (B) $x+1, y, z$. For $2:$ : (A) $x, y, z-1$. For $3:$ (A) $-x,-y+1,-z+$ 1. For 4: (A) $x+1, y$, $z$. For 5: (A) $x+2, y-1, z+1$. For 6: (A) $-x+2,-y+1,-z$; (B) $x,-y+1 / 2, z-1 / 2$. For 7: (A) $x-1,-y+3 / 2, z+1 / 2$. For 8: (A) $3 / 2-x$, $y, 2-z ;(\mathrm{B}) 1-x, 1 / 2+y, 3 / 2-z$.

and $\mathrm{SCN}^{-}$nitrogen and sulfur atoms $(\mathrm{N}(4)$ and $\mathrm{S}(1))$ generate these non-classical hydrogen bonds $(\mathrm{C}(4)-\mathrm{H}(4) \cdots \mathrm{N}(4), 3.448(6)$ $\AA$; $\mathrm{C}(8)-\mathrm{H}(8 \mathrm{~B}) \cdots \mathrm{S}(1), 3.652(5) \AA$ A), which further stabilize the $2 \mathrm{D}$ coordination polymer $1 .^{24,25}$

\section{Structure of $\left\{\left[\mathrm{Cd}\left(\mathrm{L}_{1}\right)\left(\mu_{2}-\mathrm{dca}\right)_{2}\left(\mathrm{H}_{2} \mathrm{O}\right)\right] \cdot \mathrm{H}_{2} \mathrm{O}\right\}_{n}(2)$}

The asymmetric structural unit of $\left\{\left[\mathrm{Cd}\left(\mu_{2}-\mathrm{L}_{1}\right)\left(\mu_{2}-\mathrm{dca}\right)_{2}\left(\mathrm{H}_{2} \mathrm{O}\right)\right]\right.$. $\left.\mathrm{H}_{2} \mathrm{O}\right\}_{n}(2)$ contains one central Cd ${ }^{\mathrm{II}}$ ion (Cd1), one $\mu_{2}$-bridging $\mathrm{L}_{1}$ ligand, two $\mu_{2}$-bridging dca ${ }^{-}$groups and one coordinated water 
<smiles>Cc1nnc(-c2cccnc2)o1</smiles><smiles>Cc1nnc(-c2ccncc2)o1</smiles>

Scheme 1 Two isomeric oxadiazol-pyridine ligands 3-(5-methyl1,3,4-oxadiazol-2-yl)pyridine $\left(\mathrm{L}_{1}\right)$ and 3-(5-methyl-1,3,4-oxadiazol-2yl)pyridine $\left(\mathrm{L}_{2}\right)$.

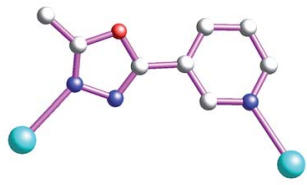

( a )

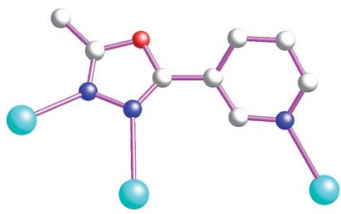

(c)

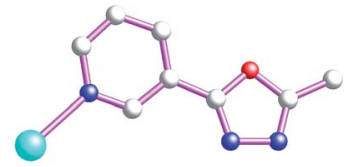

( b )

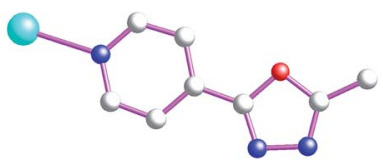

(d)

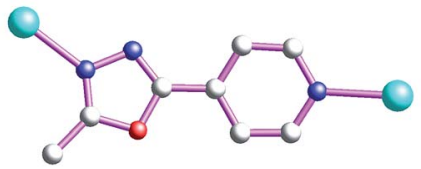

(e )

Scheme 2 Five different coordination modes of $L_{1}$ and $L_{2}$ in 1-8 (color code: cyan, metal; blue, N; light grey, C).

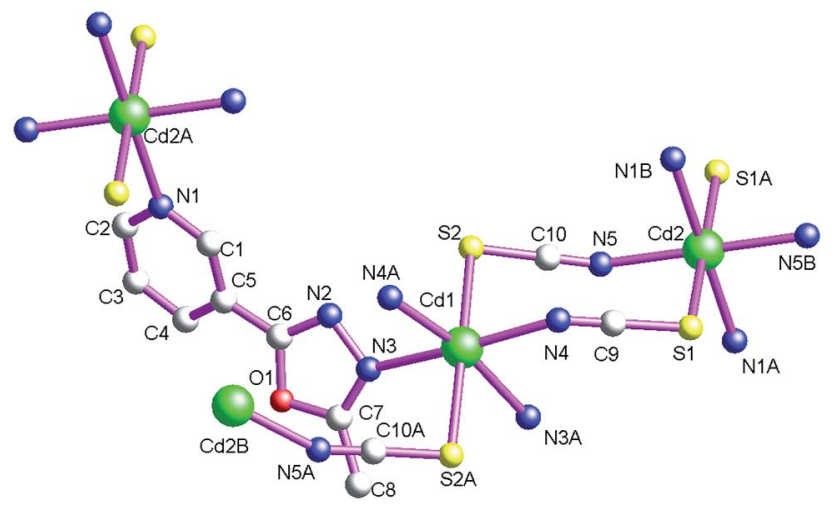

Fig. 1 The fundamental structural unit of $\left[\mathrm{Cd}_{2}\left(\mu_{2}-L_{1}\right)_{2}\left(\mu_{2}-\mathrm{NCS}\right)_{4}\right]_{n}(1)$.

molecule (O2) and one lattice aqua molecule (O3) (Fig. 3). The central $\mathrm{Cd}^{\mathrm{II}}$ atom is six-coordinated by one nitrogen atom (N1) from one $\mathrm{L}_{1}$, four $\mathrm{dca}^{-}$nitrogen atoms $(\mathrm{N} 4, \mathrm{~N} 7, \mathrm{~N} 6 \mathrm{~A}$ and $\mathrm{N} 9 \mathrm{~A})$ and one terminal aqua molecule $(\mathrm{O} 2)$ forming $\mathrm{CdN}_{5} \mathrm{O}$ donor set. The $\mathrm{Cd}-\mathrm{N}$ distances are 2.261(3)-2.326(4) $\mathrm{A}$, Cd-O distances are 2.363(2) $\AA$. All the $\mathrm{N}-\mathrm{Cd}-\mathrm{N}$ and $\mathrm{N}-\mathrm{Cd}-\mathrm{O}$ angles fall in the range of $84.10(11)-98.40(13)^{\circ}$, such coordination mode is in accordance with an octahedrally coordinated $\mathrm{Cd}^{\mathrm{II}}$ center.
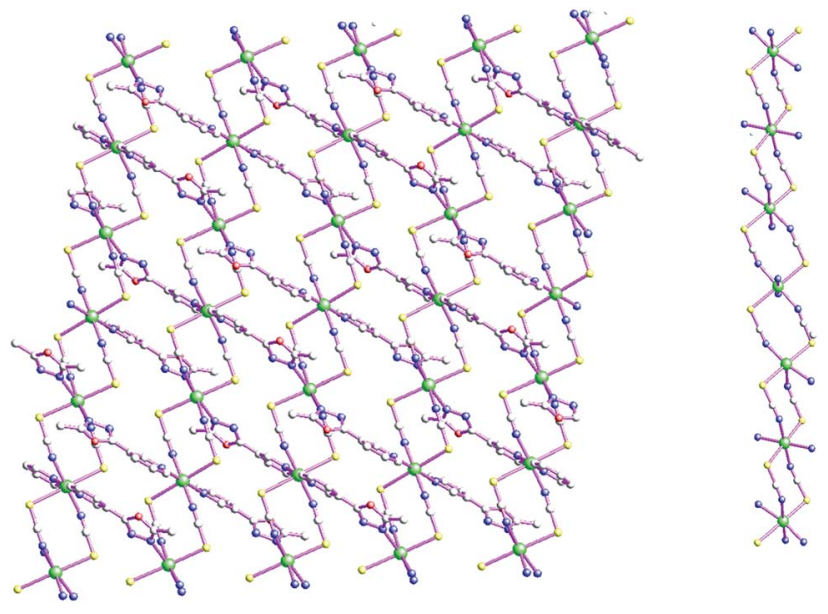

Fig. 2 2D coordination polymer $\left[\mathrm{Cd}_{2}\left(\mu_{2}-\mathrm{L}_{1}\right)_{2}\left(\mu_{2}-\mathrm{NCS}\right)_{4}\right]_{n}$ (1) containing infinite $\{\mathrm{Cd}-\mathrm{NCS}-\mathrm{Cd}\}$ chains.

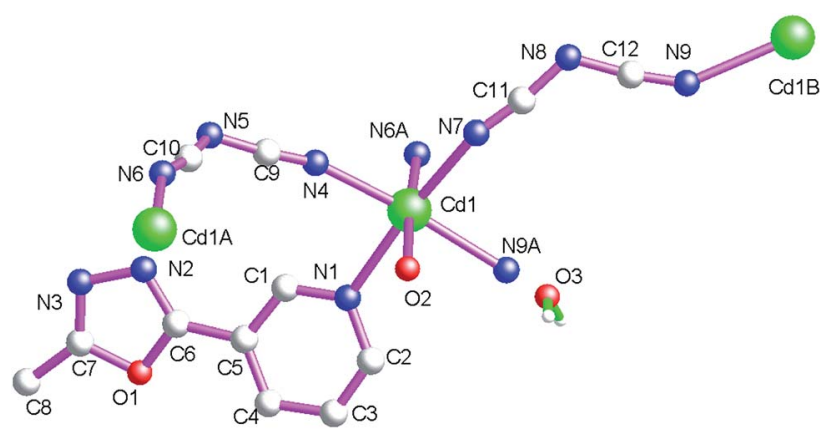

Fig. 3 The fundamental structural unit of $\left\{\left[C d\left(L_{1}\right)\left(\mu_{2}-d c a\right)_{2}\left(H_{2} O\right)\right]\right.$. $\left.\mathrm{H}_{2} \mathrm{O}\right\}_{n}(2)$.

As shown in Fig. 4, these bidentate bridging $\mathrm{dca}^{-}$ligands connect these neighboring $\mathrm{Cd}^{\mathrm{II}}$ centers forming $\{\mathrm{Cd}-\mathrm{dca}-\mathrm{Cd}\}$ layers. The shortest $\mathrm{Cd} \cdots \mathrm{Cd}$ distances within the $\{\mathrm{Cd}-\mathrm{dca}-\mathrm{Cd}\}$ layers are 7.819(1) $\AA$ and 8.575(4) $\mathrm{A}$. On the other hand, as listed in Scheme $2(\mathrm{~b})$, these $\mathrm{L}_{1}$ ligands in 2 adopt mono-dentate coordination mode. Therefore these $\{\mathrm{Cd}-\mathrm{NCS}-\mathrm{Cd}\}$ layers are further decorated via these mono-dentate $\mathrm{L}_{1}$ ligands. These coordinated aqua molecules $(\mathrm{O}(2))$, lattice aqua molecules $(\mathrm{O}(3)), \mathrm{L}_{1}$ nitrogen atoms $(\mathrm{N}(3))$ and $\mathrm{dca}^{-}$nitrogen atoms $(\mathrm{N}(8))$ generate these classical hydrogen bonds $(\mathrm{O}(2)-\mathrm{H}(2 \mathrm{~A}) \cdots \mathrm{O}(3)$, $2.740(4) \AA ; \quad \mathrm{O}(2)-\mathrm{H}(2 \mathrm{~B}) \cdots \mathrm{N}(3), 2.854(4) \AA \circ \mathrm{O}(3)-\mathrm{H}(3 \mathrm{~A}) \cdots \mathrm{O}(2)$, $2.965(4) \AA ; \mathrm{O}(3)-\mathrm{H}(3 \mathrm{~B}) \cdots \mathrm{N}(8), 2.970(6) \AA)$, which further stabilize the $2 \mathrm{D}$ coordination polymer 2 .

\section{Structure of $\left\{\left[\mathrm{Cu}\left(\mu_{2}-\mathrm{L}_{1}\right)_{2}(\mathrm{NCS})_{2}\right] \cdot 0.5 \mathrm{H}_{2} \mathrm{O}\right\}_{n}(3)$}

As shown in Fig. 5 , the asymmetric structural unit of 3 contains one central $\mathrm{Cu}^{\mathrm{II}}$ ion (Cu1), one $\mu_{2}$-bridging $\mathrm{L}_{1}$ ligand and two terminal bridging $\mathrm{SCN}^{-}$groups. The central $\mathrm{Cu}^{\mathrm{II}}$ atom is sixcoordinated by four nitrogen atoms (N1, N1A, N3A and N3B) from four $\mathrm{L}_{1}$, two thiocyanate nitrogen atoms (N4 and N4A) forming the $\mathrm{CuN}_{6}$ donor set. The $\mathrm{Cu}-\mathrm{N}$ distances are 1.974(2)2.0578(19) $\AA$, all the $\mathrm{N}-\mathrm{Cu}-\mathrm{N}$ angles fall in the range of $88.99(8)-$ 

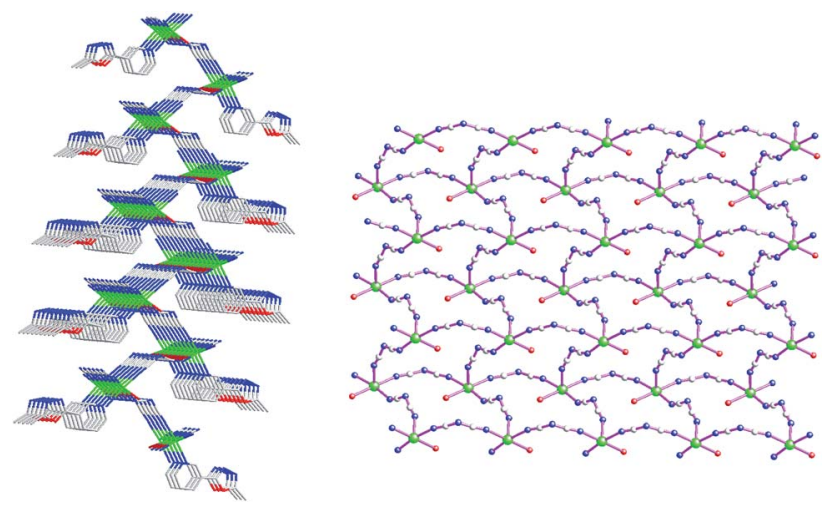

Fig. 4 Side view of $2 \mathrm{D}$ coordination polymer $\left\{\left[\mathrm{Cd}\left(\mathrm{L}_{1}\right)\left(\mu_{2}-\mathrm{dca}\right)_{2}\left(\mathrm{H}_{2} \mathrm{O}\right)\right]\right.$. $\left.\mathrm{H}_{2} \mathrm{O}\right\}_{n}(2)$ containing infinite $\{\mathrm{Cd}-\mathrm{dca}-\mathrm{Cd}\}$ layers.

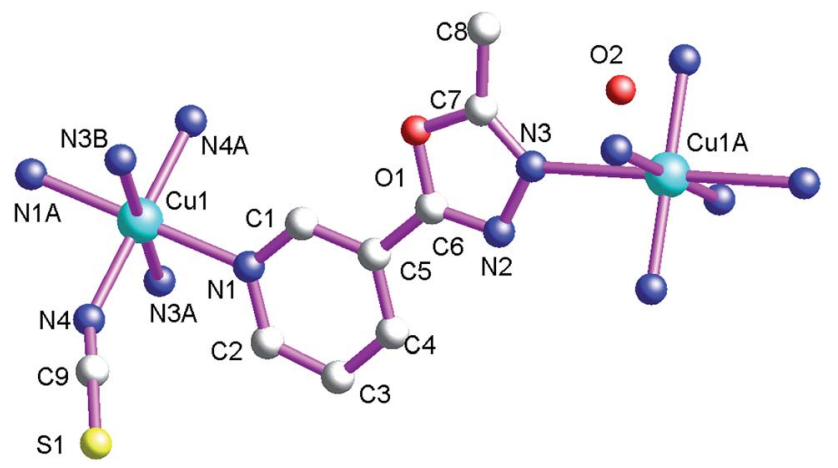

Fig. 5 The fundamental structural unit of $\left\{\left[\mathrm{Cu}\left(\mu_{2}-L_{1}\right)_{2}(\mathrm{NCS})_{2}\right]\right.$. $\left.0.5 \mathrm{H}_{2} \mathrm{O}\right\}_{n}$ (3).

91.01(8) ${ }^{\circ}$, such coordination mode is in accordance with an octahedrally coordinated $\mathrm{Cu}^{\mathrm{II}}$ center.

As shown in Fig. 6 and Scheme 2(a), these $\mathrm{L}_{1}$ ligands in 3 also adopt $\mu_{2}$-bridging coordination mode. These $\mathrm{L}_{1}$ ligands connect neighboring $\mathrm{Cu}^{\mathrm{II}}$ centers forming the $2 \mathrm{D}$ coordination polymer 3 . The corresponding $\mathrm{Cu} \cdots \mathrm{Cu}$ distance within the $2 \mathrm{D}$ coordination polymer is 9.997(5) $\AA$. These $\mathrm{L}_{1}$ carbon molecules $(\mathrm{C}(1))$ and $\mathrm{L}_{1}$

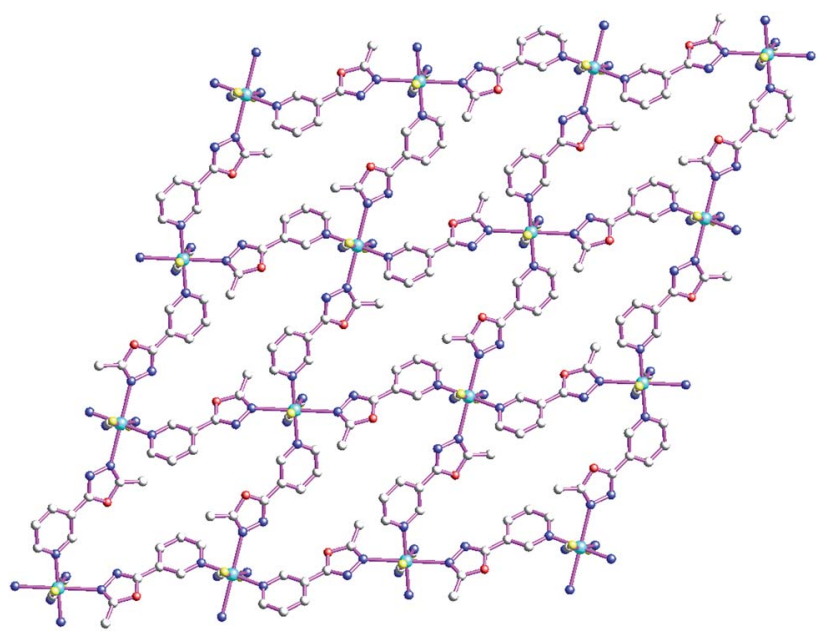

Fig. $62 \mathrm{D}$ coordination polymer $\left\{\left[\mathrm{Cu}\left(\mu_{2}-\mathrm{L}_{1}\right)_{2}(\mathrm{NCS})_{2}\right] \cdot 0.5 \mathrm{H}_{2} \mathrm{O}\right\}_{n}(3)$. nitrogen atoms $(\mathrm{N}(2))$ generate these non-classical hydrogen bonds

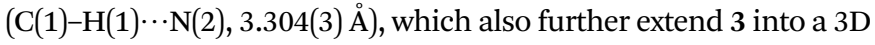
supramolecular architecture. Such weak intermolecular interactions are key for the stabilization of the 2D coordination polymer 3 .

Structure of $\left\{\left[\mathrm{Ag}_{2}\left(\mu_{2}-\mathrm{L}_{1}\right)\left(\mu_{3}-\mathrm{L}_{1}\right)_{2}\right] \cdot 2 \mathbf{P F}_{6}\right\}_{n}(4)$

When $\mathrm{AgBF}_{4}$ are used, $1 \mathrm{D} \mathrm{Ag}(\mathrm{I})$ coordination polymers, $\left\{\left[\mathrm{Ag}_{2}\left(\mu_{3}-\right.\right.\right.$ $\left.\left.\left.\mathrm{L}_{1}\right)_{2}\left(\mu_{2}-\mathrm{L}_{1}\right)\right] \cdot 2 \mathrm{PF}_{6}\right\}_{n}(\mathbf{4})$, can be isolated. For $\mathbf{4}$, the coordination polymer crystallizes in triclinic $P \overline{1}$ space group with one formula unit in the asymmetric unit. The asymmetric unit contains two $\mathrm{Ag}^{\mathrm{I}}$ atoms ( $\mathrm{Ag} 1$ and $\mathrm{Ag} 2$ ), two tri-dentate bridging $\mathrm{L}_{1}$, one bidentate bridging $\mathrm{L}_{1}$ and two free $\mathrm{PF}_{6}{ }^{-}$anions (Fig. 7). The central $\mathrm{Ag}^{\mathrm{I}}$ atom ( $\mathrm{Ag} 1$ ) is four-coordinated by four nitrogen atoms (N2, N4, N3A and N4A) from four $\mathrm{L}_{1}$ forming $\mathrm{AgN}_{4}$ donor set. The other central $\mathrm{Ag}^{\mathrm{I}}$ atom (Ag2) is also four-coordinated by four nitrogen atoms (N1, N6, N7 and N9A) from four $\mathrm{L}_{1}$ forming $\mathrm{AgN}_{4}$ donor set. The Ag-N distances are 2.225(4)-2.554(4) $\AA$, all the $\mathrm{N}-\mathrm{Ag}-\mathrm{N}$ angles are in the range of 98.30(17)-152.34(16) such coordination mode is in accord with a tetrahedrally coordinated $\mathrm{Ag}^{\mathrm{I}}$ center.

As shown in Fig. 8, Ag1 and Ag2 are inter-linked via oxadiazole nitrogen atoms forming di-nuclear Ag(I) clusters, neighboring $\mathrm{Ag} \cdots \mathrm{Ag}$ distances within di-nuclear $\mathrm{Ag}(\mathrm{I})$ clusters are 3.845(6) Å. These bridging $\mathrm{L}_{1}$ ligands connect adjacent $\mathrm{Ag}^{\mathrm{I}}$ ions forming a cluster-based 1D double chain coordination polymer, which is shown along the crystallographic $a$-axis. These nonclassical hydrogen bonds $(\mathrm{C}(8)-\mathrm{H}(8 \mathrm{~B}) \cdots \mathrm{F}(12), 3.341(9) \AA$; $\mathrm{C}(17)-\mathrm{H}(17) \cdots \mathrm{F}(7), 3.190(10) \AA$; $\mathrm{C}(17)-\mathrm{H}(17) \cdots \mathrm{F}(8), 3.347(10) \AA$; $\mathrm{C}(21)-\mathrm{H}(21) \cdots \mathrm{N}(5), 3.504(8) \AA)$ also can be observed, which further extend 4 into a 3D supramolecular architecture. Such weak intermolecular interactions are important for the stabilization of the 1D cluster-based $\mathrm{Ag}^{\mathrm{I}}$ coordination polymer 4 .

\section{Structure of $\left\{\left[\mathrm{Ag}_{3}\left(\mu_{2}-\mathrm{L}_{1}\right)_{4}\left(\mu_{2}-\mathrm{CF}_{3} \mathrm{SO}_{3}\right)\left(\mathrm{CF}_{3} \mathrm{SO}_{3}\right)\right] \cdot \mathrm{CF}_{3} \mathrm{SO}_{3}\right\}$ (5)}

When $\mathrm{AgCF}_{3} \mathrm{SO}_{3}$ is used, 1D $\mathrm{Ag}(\mathrm{I})$ coordination polymer, $\left\{\left[\mathrm{Ag}_{3}\left(\mu_{2}-\mathrm{L}_{1}\right)_{4}\left(\mu_{2}-\mathrm{CF}_{3} \mathrm{SO}_{3}\right)\left(\mathrm{CF}_{3} \mathrm{SO}_{3}\right)\right] \cdot \mathrm{CF}_{3} \mathrm{SO}_{3}\right\}$ (5), can be isolated.

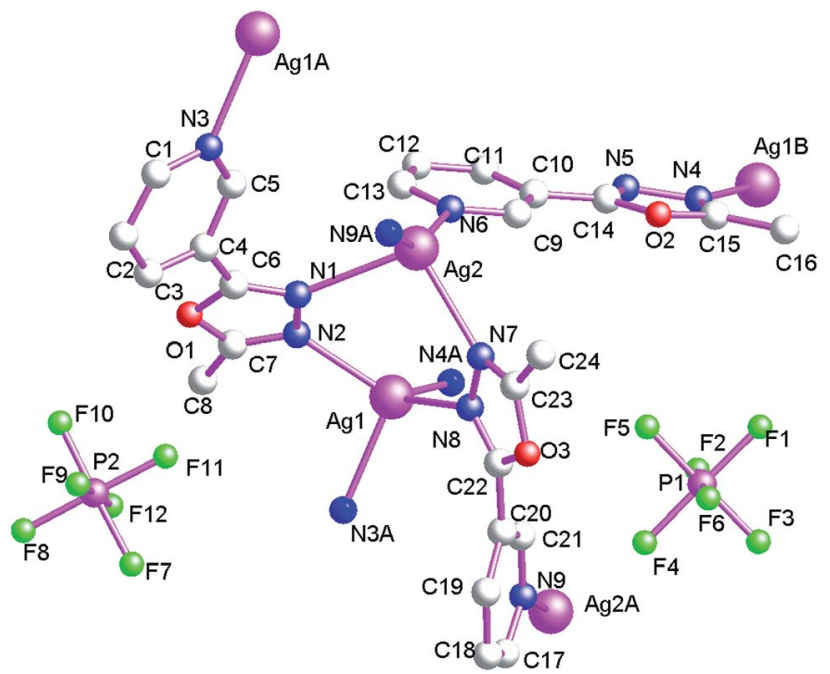

Fig. 7 The fundamental structural unit of $\left\{\left[\mathrm{Ag}_{2}\left(\mu_{2}-L_{1}\right)\left(\mu_{3}-L_{1}\right)_{2}\right] \cdot 2 P F_{6}\right\}_{n}$ (4). 

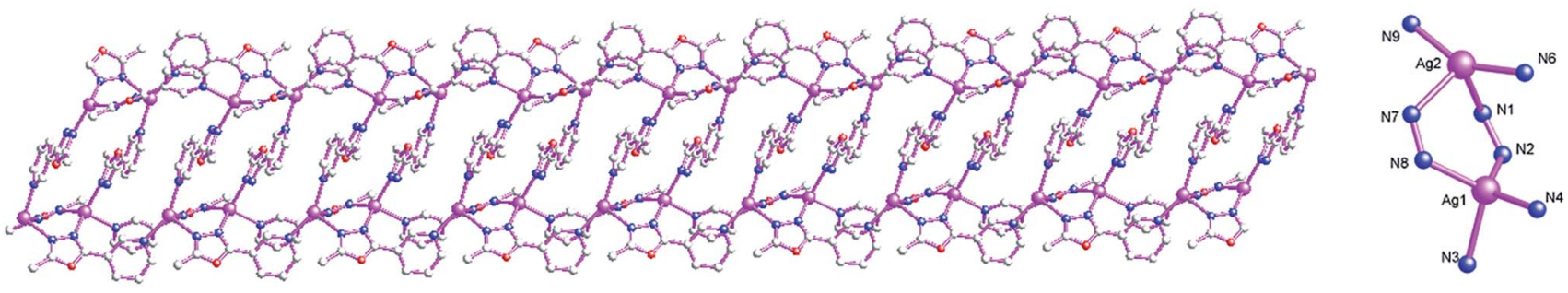

Fig. $81 \mathrm{D} \mathrm{Ag}$ ' coordination polymer $\left\{\left[\mathrm{Ag}_{2}\left(\mu_{2}-L_{1}\right)\left(\mu_{3}-L_{1}\right)_{2}\right] \cdot 2 P F_{6}\right\}_{n}(4)$.

For $\mathbf{5}$, the coordination polymer also crystallizes in triclinic $P \overline{\mathbf{1}}$ space group with one formula unit in the asymmetric unit. The asymmetric unit contains three $\mathrm{Ag}^{\mathrm{I}}$ atoms ( $\mathrm{Ag} 1, \mathrm{Ag} 2$ and $\mathrm{Ag} 3$ ), four bi-dentate bridging $\mathrm{L}_{1}$, two terminal $\mathrm{CF}_{3} \mathrm{SO}_{3}{ }^{-}$anions and one free $\mathrm{CF}_{3} \mathrm{SO}_{3}{ }^{-}$anion (Fig. 9). The central $\mathrm{Ag}^{\mathrm{I}}$ atom ( $\mathrm{Ag} 1$ ) is three-coordinated by four nitrogen atoms (N4, N1 and N12A) from three $\mathrm{L}_{1}$ forming $\mathrm{AgN}_{3}$ donor set. The central $\mathrm{Ag}^{\mathrm{I}}$ atom (Ag2) is four-coordinated by three nitrogen atoms (N3, N6 and N7) from three $\mathrm{L}_{1}$ and one $\mathrm{CF}_{3} \mathrm{SO}_{3}{ }^{-}$oxygen atom (O5) forming $\mathrm{AgN}_{3} \mathrm{O}$ donor set. The central $\mathrm{Ag}^{\mathrm{I}}$ atom (Ag3) is threecoordinated by two nitrogen atoms ( $\mathrm{N} 9$ and $\mathrm{N} 10$ ) from two $\mathrm{L}_{1}$ and one $\mathrm{CF}_{3} \mathrm{SO}_{3}{ }^{-}$oxygen atom (O9) forming $\mathrm{AgN}_{2} \mathrm{O}$ donor set. The $\mathrm{Ag}-\mathrm{N}$ distances vary from 2.209(4) $\AA$ to 2.458(4) $\AA$, Ag-O distances are 2.591(4) $\AA$, all the $\mathrm{N}-\mathrm{Ag}-\mathrm{N}$ and $\mathrm{N}-\mathrm{Ag}-\mathrm{O}$ angles fall in the range of $77.79(13)-143.19(14)^{\circ}$, All the bond distances and bond angles all fall into the normal range.

As shown in Fig. 10, $\mathrm{L}_{1}$ adopt bi-dentate bridging coordination mode, which connect neighboring Ag1, Ag2 and Ag3, forming 1D double chain coordination polymer 5 . The shortest neighboring $\mathrm{Ag} \cdots \mathrm{Ag}$ distances within 1D double chain are 4.478(2) and $4.858(8) \AA$. These non-classical hydrogen bonds $(\mathrm{C}(1)-\mathrm{H}(1 \mathrm{~A}) \cdots$

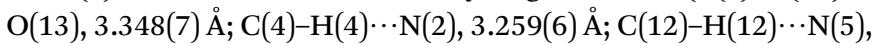
3.241(6) $\AA \mathrm{C}(15)-\mathrm{H}(15) \cdots \mathrm{O}(11), 3.393(7) \AA)$ also can be observed,

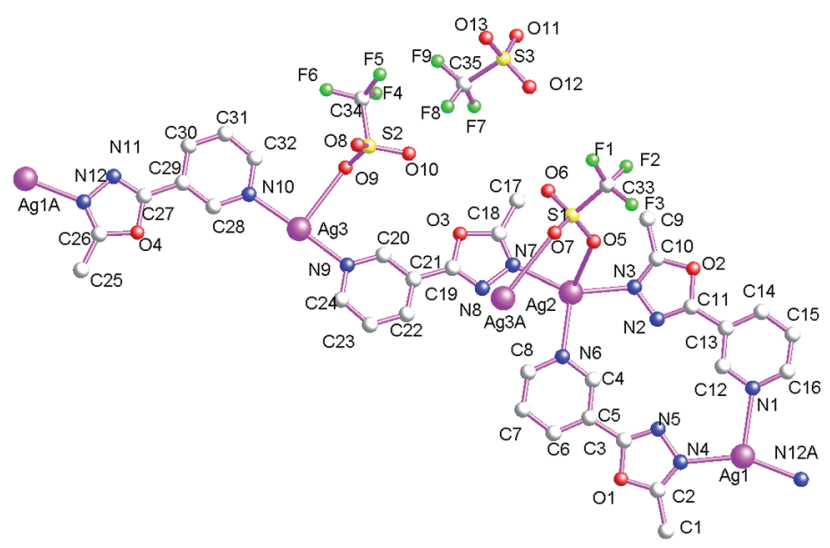

Fig. 9 The fundamental structural unit of $\left\{\left[\mathrm{Ag}_{3}\left(\mu_{2}-\mathrm{L}_{1}\right)_{4}\left(\mathrm{CF}_{3} \mathrm{SO}_{3}\right)_{2}\right]\right.$. $\left.\mathrm{CF}_{3} \mathrm{SO}_{3}\right\}(5)$. which further extend 5 into a 3D supramolecular architecture. Such weak intermolecular interactions are key for the stabilization of $1 \mathrm{D} \mathrm{Ag}{ }^{\mathrm{I}}$ double chain coordination polymer 5 .

\section{Structure of $\left\{\left[\operatorname{Cd}\left(\mathrm{L}_{2}\right)_{2}\left(\mu_{2}-\mathrm{NCS}\right)_{2}\right]\right\}_{n}(6)$}

As shown in Fig. 11, the asymmetric structural unit of $\left\{\left[\mathrm{Cd}\left(\mathrm{L}_{2}\right)_{2}\left(\mu_{2}-\mathrm{NCS}\right)_{2}\right]\right\}_{n}(6)$ contains one central $\mathrm{Cd}^{\mathrm{II}}$ ions $(\mathrm{Cd} 1)$, two terminal coordinated $\mathrm{L}_{1}$ ligands and two $\mu_{2}$-bridging $\mathrm{NCS}^{-}$ groups. The central $\mathrm{Cd}^{\mathrm{II}}$ atom is six-coordinated by two nitrogen atoms (N1 and N1A) from two $\mathrm{L}_{2}$, two nitrogen atoms (N4 and $\mathrm{N} 4 \mathrm{~A}$ ) and two sulfur atoms (S1A and S1B) form four $\mathrm{NCS}^{-}$ groups forming $\mathrm{CdN}_{4} \mathrm{~S}_{2}$ donor set. The $\mathrm{Cd}-\mathrm{N}$ distances vary from 2.325(3) to 2.364(2) $\AA$, Cd-S distances are 2.7253(9) $\AA$. All the $\mathrm{N}-\mathrm{Cd}-\mathrm{N}$ and $\mathrm{N}-\mathrm{Cd}-\mathrm{O}$ angles are in the range of 89.63(6)$91.75(8)^{\circ}$, such coordination mode is in accordance with an octahedrally coordinated $\mathrm{Cd}^{\mathrm{II}}$ center.

As shown in Fig. 12, these bidentate bridging $\mathrm{NCS}^{-}$ligands connect neighboring $\mathrm{Cd}^{\mathrm{II}}$ centers forming the $\{\mathrm{Cd}-\mathrm{NCS}-\mathrm{Cd}\}$ layers. The shortest distances of $\mathrm{Cd} \cdots \mathrm{Cd}$ within the (Cd-NCSCd\} layers are 6.280(1) and 7.570(1) ̊. On the other hand, as listed in Scheme 2(b), these $\mathrm{L}_{2}$ ligands in 6 adopt mono-dentate coordination mode. Therefore these $\{\mathrm{Cd}-\mathrm{NCS}-\mathrm{Cd}\}$ layers are

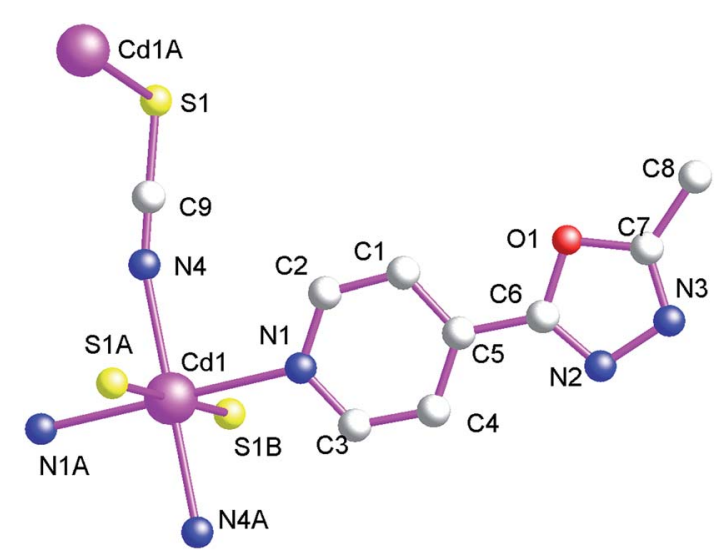

Fig. 11 The fundamental structural unit of $\left\{\left[C d\left(\mu_{2}-L_{2}\right)_{2}\left(\mu_{2}-N C S\right)_{2}\right]\right\}_{n}(6)$.

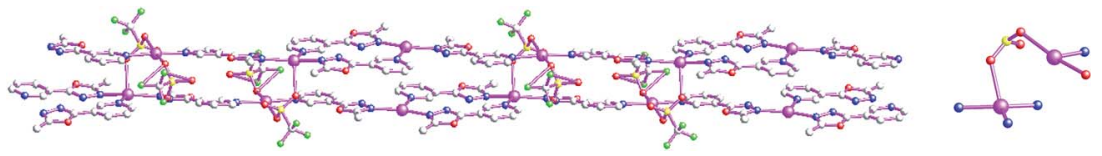

Fig. $101 \mathrm{D} \mathrm{Ag}$ coordination polymer $\left\{\left[\mathrm{Ag}_{3}\left(\mu_{2}-\mathrm{L}_{1}\right)_{4}\left(\mu_{2}-\mathrm{CF}_{3} \mathrm{SO}_{3}\right)\left(\mathrm{CF}_{3} \mathrm{SO}_{3}\right)\right] \cdot \mathrm{CF}_{3} \mathrm{SO}_{3}\right\}(5)$. 

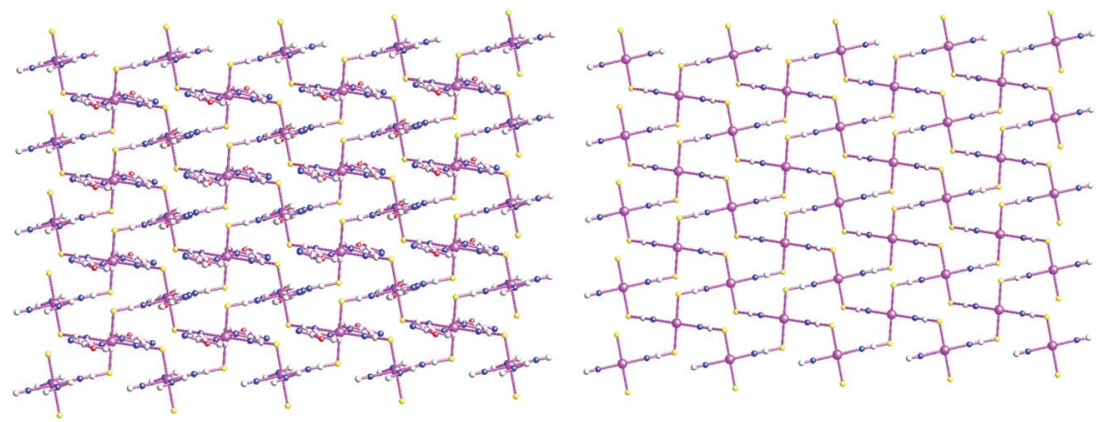

Fig. 12 2D coordination polymer $\left\{\left[C d\left(\mu_{2}-L_{2}\right)_{2}\left(\mu_{2}-N C S\right)_{2}\right]\right\}_{n}(6)$ containing infinite $\{C d-N C S-C d\}$ layers.

further decorated via these mono-dentate $\mathrm{L}_{2}$ ligands. These $\mathrm{L}_{2}$ carbon atoms $(\mathrm{C}(1))$ and nitrogen atoms $(\mathrm{N}(3))$ generate nonclassical hydrogen bonds $(\mathrm{C}(1)-\mathrm{H}(1) \cdots \mathrm{N}(3), 3.528(4) \AA)$, which further stabilize the $2 \mathrm{D}$ coordination polymer 6 .

\section{Structure of $\left[\mathrm{Ag}\left(\mu_{2}-\mathrm{L}_{2}\right) \cdot\left(\mu_{2}-\mathrm{CF}_{3} \mathrm{SO}_{3}\right)\right]_{n}(7)$}

As shown in Fig. 13, the asymmetric structural unit of $\left[\mathrm{Ag}\left(\mu_{2}-\right.\right.$ $\left.\left.\mathrm{L}_{2}\right) \cdot\left(\mu_{2}-\mathrm{CF}_{3} \mathrm{SO}_{3}\right)\right]_{n}(7)$ contains one central $\mathrm{Ag}^{\mathrm{I}}$ ions (Ag1), one $\mu_{2^{-}}$ bridging $\mathrm{L}_{2}$ ligand, one $\mu_{2}$-bridging $\mathrm{CF}_{3} \mathrm{SO}_{3}{ }^{-}$group. For 7 , the coordination polymer crystallizes in monoclinic $P 2_{1} / n$ space group with one formula unit in the asymmetric unit. The central $\mathrm{Ag}^{\mathrm{I}}$ atom (Ag1) is four-coordinated by two nitrogen atoms (N1 and $\mathrm{N} 3 \mathrm{~A}$ ) from two $\mathrm{L}_{1}$ and two $\mathrm{CF}_{3} \mathrm{SO}_{3}{ }^{-}$oxygen atoms (O2A and O4) forming $\mathrm{AgN}_{2} \mathrm{O}_{2}$ donor set. $\mathrm{Ag}-\mathrm{N}$ distances are 2.165(2)2.184(2) $\AA$, Ag-O distances are 2.623(2)-2.710(2) $\AA$, all the N-Ag$\mathrm{N}$ and $\mathrm{N}-\mathrm{Ag}-\mathrm{O}$ angles are in the range of $83.54(1)-94.19(1)^{\circ}$, such coordination mode is in accord with a tetrahedrally coordinated $\mathrm{Ag}^{\mathrm{I}}$ center.

As shown in Fig. 14, these $\mathrm{CF}_{3} \mathrm{SO}_{3}{ }^{-}$anions adopt bi-dentate bridging coordination modes via its two oxygen atoms $(\mathrm{O} 2$ and O4). On the other hand, these $\mathrm{L}_{2}$ ligands also adopt bridging coordination mode as listed in Scheme 2(e). These bridging $\mathrm{CF}_{3} \mathrm{SO}_{3}{ }^{-}$anions and $\mathrm{L}_{2}$ ligands link these neighboring $\mathrm{Ag}(\mathrm{I})$ centers, which ultimately forming the $2 \mathrm{D}$ coordination polymer 7. These $\mathrm{L}_{2}$ carbon atoms $(\mathrm{C}(2)$ and $\mathrm{C}(6))$ and $\mathrm{CF}_{3} \mathrm{SO}_{3}{ }^{-}$oxygen and fluoride atoms $(\mathrm{O}(4)$ and $\mathrm{F}(1))$ generate these non-classical

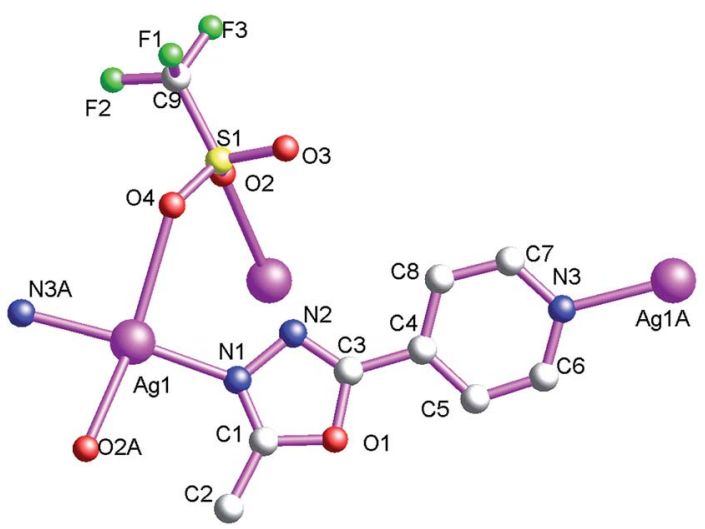

Fig. 13 The fundamental structural unit of $\left[\mathrm{Ag}\left(\mu_{2}-\mathrm{L}_{2}\right)\left(\mu_{2}-\mathrm{CF}_{3} \mathrm{SO}_{3}\right)\right]_{n}(7)$.

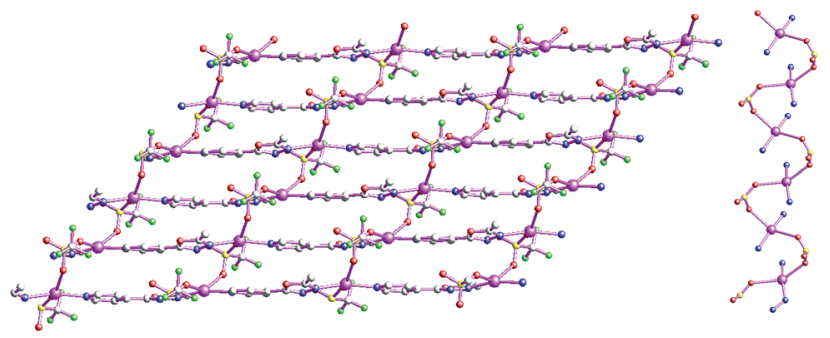

Fig. $142 \mathrm{D}$ coordination polymer $\left[\mathrm{Ag}\left(\mu_{2}-\mathrm{L}_{2}\right)\left(\mu_{2}-\mathrm{CF}_{3} \mathrm{SO}_{3}\right)\right]_{n}$ (7).

hydrogen bonds (C(2)-H(2C) $\cdots \mathrm{F}(1), 3.338(5) \AA ; \mathrm{C}(6)-\mathrm{H}(6) \cdots \mathrm{O}(4)$, $3.308 \AA$ ), which further extend 7 into a 3D supramolecular architecture. Such weak intermolecular interactions are important for the stabilization of the $1 \mathrm{D}$ cluster-based $\mathrm{Ag}^{\mathrm{I}}$ coordination polymer 7 .

\section{Structure of $\left\{\left[\operatorname{Ag}\left(\mu_{2}-\mathrm{L}_{2}\right)\right] \cdot \mathrm{BF}_{4}\right\}_{n}(8)$}

As shown in Fig. 15, the fundamental structural unit of $\left\{\left[\operatorname{Ag}\left(\mu_{2}-\right.\right.\right.$ $\left.\left.\left.\mathrm{L}_{2}\right)\right] \cdot \mathrm{BF}_{4}\right\}_{n}$ (9) contains one central $\mathrm{Ag}^{\mathrm{I}}$ ions (Ag1), one $\mu_{2}$ bridging $\mathrm{L}_{2}$ ligand, one free $\mathrm{BF}_{4}{ }^{-}$group. For 8, the coordination polymer crystallizes in monoclinic $I_{2} / a$ space group with one formula unit in the asymmetric unit. The central $\mathrm{Ag}^{\mathrm{I}}$ atom (Ag1) is four-coordinated by four nitrogen atoms $(\mathrm{N} 1, \mathrm{~N} 3 \mathrm{~A}, \mathrm{~N} 1 \mathrm{~A}$ and $\mathrm{N} 3 \mathrm{~B}$ ) from four $\mathrm{L}_{2}$ forming $\mathrm{AgN}_{4}$ donor set. The $\mathrm{Ag}-\mathrm{N}$ distances are 2.282(3)-2.349(3) $\AA$, all the $\mathrm{N}-\mathrm{Ag}-\mathrm{N}$ angles are in the range of 94.65(17)-125.45(12) ${ }^{\circ}$, such coordination mode is in accord with a tetrahedrally coordinated $\mathrm{Ag}^{\mathrm{I}}$ center.

As shown in Fig. 16, these $\mathrm{L}_{2}$ ligands also adopt bridging coordination mode as listed in Scheme 2(e). These bridging $\mathrm{L}_{2}$

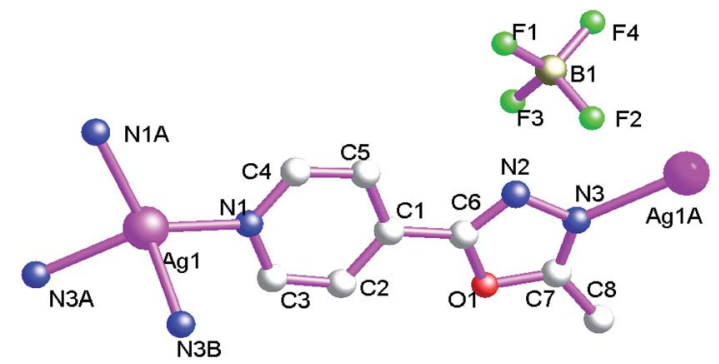

Fig. 15 The fundamental structural unit of $\left\{\left[\mathrm{Ag}\left(\mu_{2}-L_{2}\right)\right] \cdot B F_{4}\right\}_{n}(8)$. 


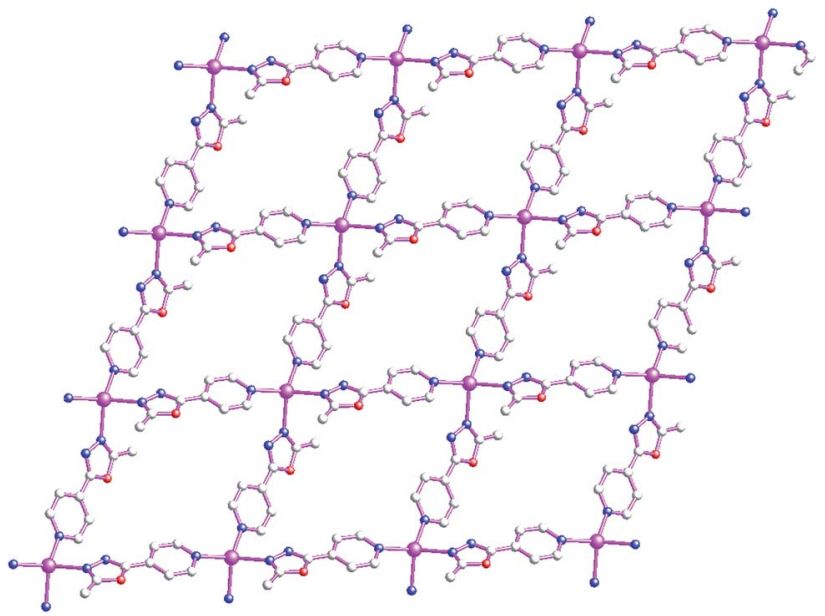

Fig. $162 \mathrm{D}$ cationic $A g^{\prime}$ coordination polymer $\left\{\left[A g\left(\mu_{2}-L_{2}\right)\right] \cdot B F_{4}\right\}_{n}(8)$.

ligands link these neighboring $\mathrm{Ag}(\mathrm{I})$ centers, which ultimately forming the $2 \mathrm{D}$ grid-like cationic coordination polymer. These non-classical hydrogen bonds $(\mathrm{C}(4)-\mathrm{H}(4) \cdots \mathrm{F}(2), 3.085(9) \AA)$ also can be observed, which further extend 8 into a 3D supramolecular architecture. Such weak intermolecular interactions are important for the stabilization of the $2 \mathrm{D} \mathrm{Ag}^{\mathrm{I}}$ coordination polymer.

\section{Magnetic properties of 3}

Variable temperature dc magnetic susceptibility data were collected on a microcrystalline powder sample of 3 in a $1000 \mathrm{Oe}$ field in the temperature range $1.8-300 \mathrm{~K}$. The temperaturedependent magnetic susceptibility data of complexes 3 have been measured in the temperature range of $2-300 \mathrm{~K}$. The diamagnetic correction was evaluated by using Pascal's constants. As shown in Fig. 17, The $\chi_{\mathrm{m}} T$ values are $c a .0 .416 \mathrm{~cm}^{3}$ $\mathrm{kmol}^{-1}$ (for 3) at room temperature, which are expected spinonly values for an isolated $\mathrm{Cu}$ (II) ion. When the temperature is lowered, $\chi_{\mathrm{M}} T$ values remain constants and then smoothly decrease and drop rapidly below $c a .20 \mathrm{~K}$ reaching the

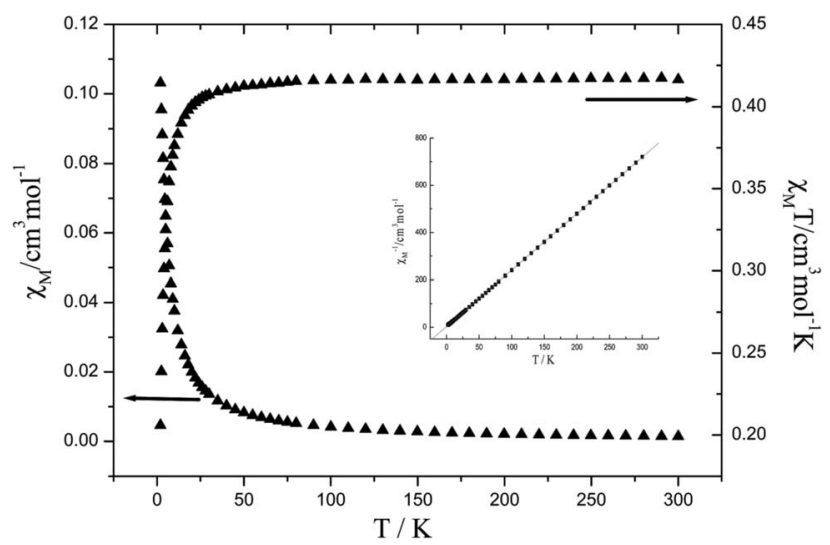

Fig. 17 Variable-temperature magnetic susceptibility data for 3 in the 2-300 K temperature range. minimum values at $2 \mathrm{~K}$. Fitting the magnetic susceptibility data with the Curie-Weiss law $\chi_{\mathrm{M}}=C /(T-\theta)$, where $C$ and $\theta$ represent the Curie constant and the Weiss temperature, it can give $\theta$ values of $-1.05 \mathrm{~K}$. These $\theta$ values reveal antiferromagnetic interactions between central $\mathrm{Cu}^{\mathrm{II}}$ ions. The result of weak anti-ferromagnetic interactions can be attributed to can be attributed to very weak antiferromagnetic interactions as indicated by large metal-metal distances or the presence of zero-field splitting or both in $3 .^{26}$

\section{Luminescence properties}

Coordination polymers have been investigated for potential applications as luminescent materials, such as light-emitting diodes (LEDs). ${ }^{27}$ Owing to the ability of affecting the emission wavelength and strength of organic materials, syntheses of coordination polymers by judicious choice of conjugated organic spacers and transition metal centers can be an efficient method for obtaining new types of luminescent materials, especially for $\mathrm{d}^{10}$ or $\mathrm{d}^{10}-\mathrm{d}^{10}$ systems. ${ }^{28}$ In the present work, we have explored the luminescent properties of $\mathrm{L}_{1}, \mathrm{~L}_{2}$ ligands and organic/inorganic coordination polymers $\mathbf{1 ,} \mathbf{2}$ and $\mathbf{4 - 8}$ based on the ligands in the solid state.

As shown in Fig. 18, at room temperature, free $\mathrm{L}_{1}$ and $\mathrm{L}_{2}$ ligands in the solid state are luminescent, which show the broad emission maximum centered at $360 \mathrm{~nm}\left(\lambda_{\mathrm{ex}}=280 \mathrm{~nm}\right)$ and $361 \mathrm{~nm}\left(\lambda_{\mathrm{ex}}=280 \mathrm{~nm}\right)$, respectively. For the free $\mathrm{L}_{1}$ and $\mathrm{L}_{2}$ ligands, the chromophores are aromatic pyridine and oxadiazol rings, the observed emission is due to $\pi-\pi^{*}$ transition. In comparison with that of free ligand, upon excitation of solid samples of 1, 2, 6 and 8 show emission bands with maximum at $c a .360 \mathrm{~nm}$ (for 1), $c a$. $361 \mathrm{~nm}$ (for 2), ca. $363 \mathrm{~nm}$ (for 6) and $c a .362 \mathrm{~nm}$ (for 8) respectively. The emissions of 1, 2, 6 and 8 should be assigned to intraligand $\pi-\pi^{*}$ transitions. ${ }^{29}$ The slight shifts of the emission bands relative to free $\mathrm{L}_{1}$ and $\mathrm{L}_{2}$ ligands can be attributed to the coordination action of ligand to central metal ions. ${ }^{30}$

On the other hand, for these 1D or 2D cluster-based $\mathrm{Ag}^{\mathrm{I}}$ coordination polymers $\mathbf{4 , 5}$ and 7, it is noted that red-shift of

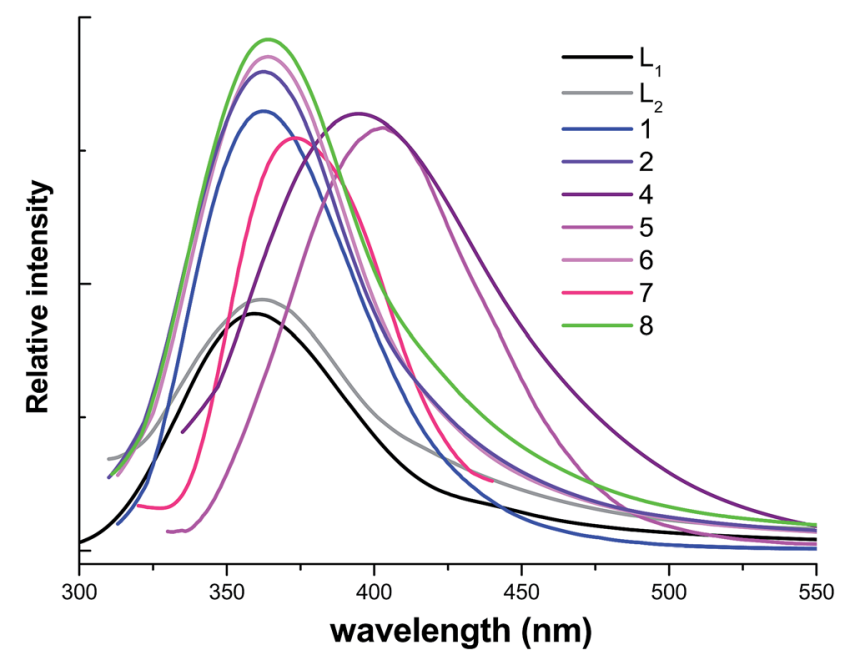

Fig. 18 Solid-state fluorescence spectra of $1-2,4-8$ and $L_{1}, L_{2}$ ligand molecules. 
luminescent emissions can be observed (emission bands with maximum centered at $395 \mathrm{~nm}$ for $\mathbf{4}\left(\lambda_{\mathrm{ex}}=300 \mathrm{~nm}\right), 403 \mathrm{~nm}$ for 5 $\left(\lambda_{\mathrm{ex}}=300 \mathrm{~nm}\right)$ and $372 \mathrm{~nm}$ for $\left.7\left(\lambda_{\mathrm{ex}}=300 \mathrm{~nm}\right)\right)$. The photoluminescence origin of the emission bands should be attributed to metal-to-ligand charge transfer (MLCT) as discussed in the previous literature. ${ }^{1}$ Because 5 and 6 contain similar 1D structural motifs, different band shapes in the luminescent emissions of 5 and 6 can be ascribed to different guest $\mathrm{PF}_{6}{ }^{-} / \mathrm{CF}_{3} \mathrm{SO}_{3}{ }^{-}$ anions, which can greatly affect luminescence emission bands. ${ }^{31}$ These cluster-based $\mathrm{Ag}^{\mathrm{I}}$ coordination polymers increase the ligand conformational rigidity, thereby reducing the non-radiative decay of the intraligand $\left(\pi-\pi^{*}\right)$ excited state. The syntheses of new $\mathrm{Cd}(\mathrm{II})$ and $\mathrm{Ag}$ (I) complexes with these isomeric oxadiazol-pyridine ligands can be an efficient method for obtaining new types of luminescent materials. ${ }^{32,33}$

\section{Luminescent sensing properties of $\mathrm{Cr}_{2} \mathrm{O}_{7}{ }^{2-}$ anion pollutants for 8}

In general, water pollutions are a global environmental issue, and considerable research attention has been paid to the removal of pollutants from waste water. With the development of modern industry, $\mathrm{Cr}_{2} \mathrm{O}_{7}{ }^{2-}$ anions have been widely used in the field of chromium plating, metallurgy, pigment manufacturing, leather tanning, and wood preservation. These anion pollutants have been a focus of concern because they cause serious damage to human health and the environment. Therefore, the exploration of new materials for the efficient detection of these anion pollutants is highly important. ${ }^{34}$

In this work, the product of $\mathbf{8}$ as the luminescent probes was carefully explored for sensing diverse ions, owing to its strong visible blue light when excited by ultraviolet light. The photoluminescence emission bands of $\mathbf{8}$ in aqueous solutions are centered at around $360 \mathrm{~nm}$. These emission bands should be more likely to be due to intra-ligand transitions because similar photo-luminescent bands can be observed for free $\mathrm{L}_{2}$ ligands in aqueous solutions. On the other hand, water stability of 8 was also investigated, the solid state samples were suspended in aqueous solutions for $12 \mathrm{~h}$, then water solutions were filtrated and solid state samples were dried by the vacuum oven. Then solid state samples were characterized by PXRD patterns. As shown in Fig. S1, $\uparrow$ PXRD patterns of solid state samples suspended in the water solutions and these calculated patterns deduced from single-crystal X-ray data are in good agreement. The result indicates that the coordination polymer structure is unchangeable and can retain stable in aqueous solutions.

In order to study the luminescent responses to different anions, the products of $\mathbf{8}$ were ground into powder and suspended in the water solution containing different salt solutions of the same concentration $\left(10^{-4} \mathrm{M}\right)$ of $\mathrm{SiF}_{6}{ }^{2-}, \mathrm{CO}_{3}{ }^{2-}, \mathrm{HCO}_{3}{ }^{2-}$, $\mathrm{BF}_{4}{ }^{-}, \mathrm{NO}_{3}{ }^{-}, \mathrm{SCN}^{-}, \mathrm{Ac}^{-}, \mathrm{SO}_{4}{ }^{2-}, \mathrm{Br}^{-}, \mathrm{Cl}^{-}, \mathrm{F}^{-}, \mathrm{I}^{-}, \mathrm{BrO}_{3}{ }^{-}, \mathrm{IO}_{3}{ }^{-}$, $\mathrm{CrO}_{4}{ }^{2-}, \mathrm{HPO}_{4}{ }^{2-}$ and $\mathrm{PO}_{4}{ }^{3-}$ and $\mathrm{Cr}_{2} \mathrm{O}_{7}{ }^{2-}$. Aqueous solutions of $\mathrm{SiF}_{6}{ }^{2-}, \mathrm{CO}_{3}{ }^{2-}, \mathrm{HCO}_{3}{ }^{2-}, \mathrm{BF}_{4}{ }^{-}, \mathrm{NO}_{3}{ }^{-}, \mathrm{SCN}^{-}, \mathrm{Ac}^{-}, \mathrm{SO}_{4}{ }^{2-}, \mathrm{Br}^{-}$, $\mathrm{Cl}^{-}, \mathrm{F}^{-}, \mathrm{I}^{-}, \mathrm{BrO}_{3}{ }^{-}, \mathrm{IO}_{3}{ }^{-}, \mathrm{CrO}_{4}{ }^{2-}, \mathrm{HPO}_{4}{ }^{2-}$ and $\mathrm{PO}_{4}{ }^{3-}$ and $\mathrm{Cr}_{2} \mathrm{O}_{7}{ }^{2-}$ were prepared from these corresponding potassium salts, respectively. As shown in Fig. 19(a), $\mathrm{Cr}_{2} \mathrm{O}_{7}{ }^{2-}$ gave significant fluorescence quenching effect, while there was only a negligible effect on the luminescence intensity for other anions. PXRD pattern of $8-\mathrm{Cr}_{2} \mathrm{O}_{7}{ }^{2-}$ was measured and remains well consistent with the simulated one of $\mathbf{8}$, indicating that $\mathbf{8}$ still remains stable. The result indicates the high selectivity of 8 for the detection and specific recognition of $\mathrm{Cr}_{2} \mathrm{O}_{7}{ }^{2-}$ anions in aqueous solutions. Therefore $\mathbf{8}$ may be chosen as a candidate for the selective sensing of $\mathrm{Cr}_{2} \mathrm{O}_{7}{ }^{2-}$.

Generally, waste water contains more than one type of pollutant anions, and therefore, it is essential to investigate the influence of mixed anions on the luminescence of 8. The detailed experiments are as follows: $0.4 \mathrm{~mL}$ of $\mathrm{Na}_{2} \mathrm{Cr}_{2} \mathrm{O}_{7}\left(10^{-4}\right.$ $\mathrm{M})$ and $0.4 \mathrm{~mL}$ of other anion solutions $\left(10^{-4} \mathrm{M}\right)$ were slowly dropped into a $3.2 \mathrm{~mL}$ suspension of $\mathbf{8}$, respectively. The luminescence measurements of resultant solutions, containing $\mathrm{Cr}_{2} \mathrm{O}_{7}{ }^{2-}$ and other anions, were carried out at once. It is also noted that there was only a negligible effect on the luminescence intensity for $\mathrm{Cr}_{2} \mathrm{O}_{7}{ }^{2-}$ anions. This selective detection of $\mathrm{Cr}_{2} \mathrm{O}_{7}{ }^{2-}$ anions has no interfering effects from other anions such as $\mathrm{SiF}_{6}{ }^{2-}, \mathrm{CO}_{3}{ }^{2-}, \mathrm{HCO}_{3}{ }^{2-}, \mathrm{BF}_{4}{ }^{-}, \mathrm{NO}_{3}{ }^{-}, \mathrm{SCN}^{-}, \mathrm{Ac}^{-}, \mathrm{SO}_{4}{ }^{2-}$, $\mathrm{Br}^{-}, \mathrm{Cl}^{-}, \mathrm{F}^{-}, \mathrm{I}^{-}, \mathrm{BrO}_{3}{ }^{-}, \mathrm{IO}_{3}{ }^{-}, \mathrm{CrO}_{4}{ }^{2-}, \mathrm{HPO}_{4}{ }^{2-}$ and $\mathrm{PO}_{4}{ }^{3-}$, suggesting that 8 can selectively detect $\mathrm{Cr}_{2} \mathrm{O}_{7}{ }^{2-}$ anions among the above anions.

Moreover, to explore the detection limit of $\mathbf{8}$ as a luminescent probe for detecting $\mathrm{Cr}_{2} \mathrm{O}_{7}{ }^{2-}$, as shown in Fig. 19(b), a series suspension of $8-\mathrm{Cr}_{2} \mathrm{O}_{7}{ }^{2-}(5 \mu \mathrm{M}$ to $100 \mu \mathrm{M})$ were prepared by dropping different concentrations of $\mathrm{Cr}_{2} \mathrm{O}_{7}{ }^{2-}$ solutions into the suspension of $\mathbf{8}$. The luminescence intensity of $\mathbf{8}$ gradually decreases with increasing the concentration of $\mathrm{Cr}_{2} \mathrm{O}_{7}{ }^{2-}$. As shown in Fig. 19(c) and (d), the luminescence intensity linearly decreases with the concentration of $\mathrm{Cr}_{2} \mathrm{O}_{7}{ }^{2-}$ ranging from 0.5 $\mu \mathrm{M}$ to $70 \mu \mathrm{M}$. The detection limit of 8 as a luminescent probe for detecting $\mathrm{Cr}_{2} \mathrm{O}_{7}{ }^{2-}$ is supported by the calculated results based on the equation: detection limit $=3 \sigma / k$ ( $\sigma$ is the standard deviation of blank measurement; $k$ is the slope between the luminescence intensity $v s . \log \left[\mathrm{Cr}_{2} \mathrm{O}_{7}{ }^{2-}\right]$ ). To further explore the relationship between the quenching effect and $\mathrm{Cr}_{2} \mathrm{O}_{7}{ }^{2-}$ concentration, the linear luminescence intensity $v s . \mathrm{Cr}_{2} \mathrm{O}_{7}{ }^{2-}$ concentration plot was made, which can be fitted into $I_{0} / I=1+$ $K_{\mathrm{sv}}\left[\mathrm{Cr}_{2} \mathrm{O}_{7}{ }^{2-}\right]\left(I_{0}\right.$ and $I$ represent the luminescence intensity of $\mathbf{8}$ before and after adding $\mathrm{Cr}_{2} \mathrm{O}_{7}{ }^{2-}$, respectively; $\left[\mathrm{Cr}_{2} \mathrm{O}_{7}{ }^{2-}\right]$ represents the concentration of $\mathrm{Cr}_{2} \mathrm{O}_{7}{ }^{2-}$, and $K_{\mathrm{sv}}$ represents the quenching rate constant). The $K_{\mathrm{sv}}$ value is calculated to be 2.08 $\times 10^{4} \mathrm{~L} \mathrm{~mol}^{-1}$, indicating the high quenching efficiency of $\mathrm{Cr}_{2} \mathrm{O}_{7}{ }^{2-}$ in the emission of $\mathbf{8}^{35-37}$ The high quenching efficiency and low detection limit $0.19 \mu \mathrm{M}(\mathrm{S} / \mathrm{N}=3)$ also reveal that 8 can act as luminescent probes for discrimination and detection of $\mathrm{Cr}_{2} \mathrm{O}_{7}{ }^{2-}$.

8 is a cationic coordination polymer, in which free $\mathrm{BF}_{4}{ }^{-}$ anions are located between these 2D cationic layers, while in other $\mathrm{Ag}^{\mathrm{I}}$ coordination polymers these anions are tightly linked to main frameworks through coordination bonds. As illustrated in the previous literature, these cationic coordination polymers can effectively capture $\mathrm{Cr}_{2} \mathrm{O}_{7}{ }^{2-}$ anions through anion exchange process, ${ }^{38}$ which may effectively lead to the fluorescent quenching of 8. On the other hand, in comparison with other anions, only $\mathrm{Cr}_{2} \mathrm{O}_{7}{ }^{2-}$ exhibits two wide absorption bands from 230 to $413 \mathrm{~nm} .{ }^{39}$ The bands almost cover the whole ranges of 


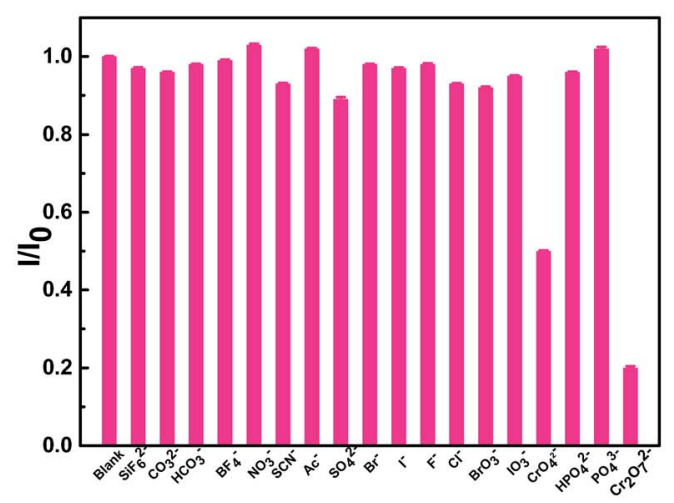

(a)

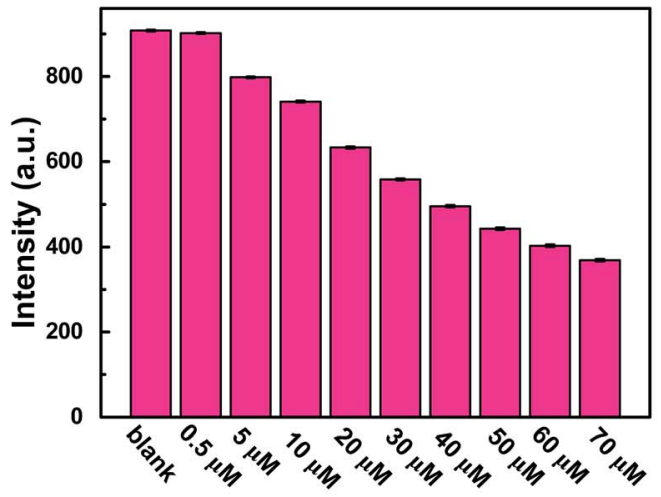

(c)

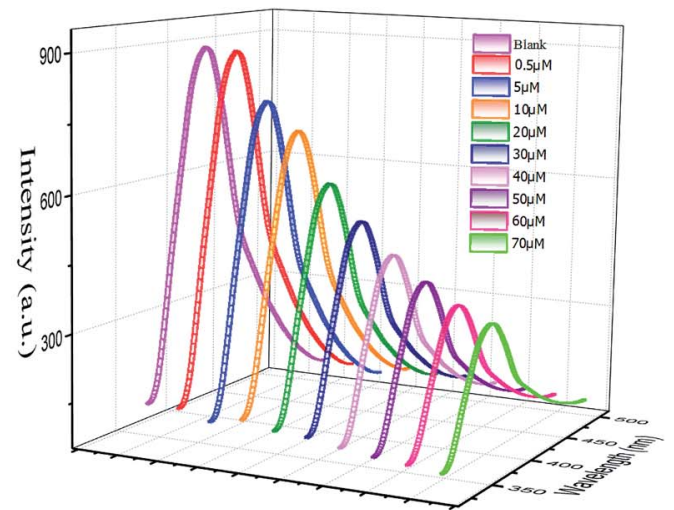

(b)

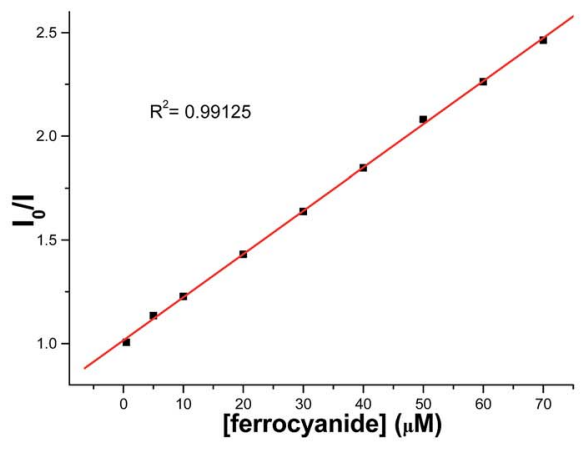

(d)

Fig. 19 (a) Comparison of the luminescence intensity of 8 at $360 \mathrm{~nm}$ incorporating aqua solutions $\left(10^{-4} \mathrm{M}\right)$ upon the addition of various anions; (b) liquid luminescence spectra of 8 under different concentrations of $\mathrm{Cr}_{2} \mathrm{O}_{7}{ }^{2-}$ aqueous solution; (c) comparison of the luminescence intensity of 8 under different concentrations of $\mathrm{Cr}_{2} \mathrm{O}_{7}{ }^{2-}$ anion pollutants aqueous solution. (d) The linear luminescence intensity $v s$. $\mathrm{Cr}_{2} \mathrm{O}_{7}{ }^{2-}$ anion pollutants concentration plot.

absorption bands that arise from 8 . Thus, the luminescence quenching mechanism also further causes by the competition of excitation energy between $\mathrm{Cr}_{2} \mathrm{O}_{7}{ }^{2-}$ anions and 8. Upon illumination, the adsorption of $\mathrm{Cr}_{2} \mathrm{O}_{7}{ }^{2-}$ ions for excitation energy hinders the UV/vis absorption of the target coordination polymer, thus resulting in a decrease, or even full quenching, of the luminescence intensities. This luminescent sensing mechanism for $\mathrm{Cr}_{2} \mathrm{O}_{7}{ }^{2-}$ is consistent with those previously proposed by previous literature. ${ }^{40,41}$ To the best of our knowledge, work on these metal-organic polymers as luminescent probes for discrimination and detection of $\mathrm{Cr}_{2} \mathrm{O}_{7}{ }^{2-}$ is important and still limited. ${ }^{42} 8$ also represents the first report of coordination polymers based on oxadiazol-pyridine derivatives as luminescent response to $\mathrm{Cr}_{2} \mathrm{O}_{7}{ }^{2-}$ in the water solutions.

\section{Conclusions and perspectives}

In conclusion, in this work two position-isomeric oxadiazolpyridine ligands 3-(5-methyl-1,3,4-oxadiazol-2-yl)pyridine $\left(\mathrm{L}_{1}\right)$ and 4-(5-methyl-1,3,4-oxadiazol-2-yl)pyridine $\left(\mathrm{L}_{2}\right)$ have been designed and synthesized. A series of novel coordination polymers, namely $\left[\mathrm{Cd}_{2}\left(\mu_{2}-\mathrm{L}_{1}\right)_{2}\left(\mu_{2}-\mathrm{NCS}\right)_{4}\right]_{n}(\mathbf{1}),\left\{\left[\mathrm{Cd}\left(\mathrm{L}_{1}\right)\left(\mu_{2}-\mathrm{dca}\right)_{2}\left(\mathrm{H}_{2} \mathrm{O}\right)\right]\right.$.
$\left.\mathrm{H}_{2} \mathrm{O}\right\}_{n}(2),\left\{\left[\mathrm{Cu}\left(\mu_{2}-\mathrm{L}_{1}\right)_{2}(\mathrm{NCS})_{2}\right] \cdot 0.5 \mathrm{H}_{2} \mathrm{O}\right\}_{n}(3),\left\{\left[\mathrm{Ag}_{2}\left(\mu_{2}-\mathrm{L}_{1}\right)\left(\mu_{3}-\mathrm{L}_{1}\right)_{2}\right]\right.$. $\left.2 \mathrm{PF}_{6}\right\}_{n}(4),\left\{\left[\mathrm{Ag}_{3}\left(\mu_{2}-\mathrm{L}_{1}\right)_{4}\left(\mu_{2}-\mathrm{CF}_{3} \mathrm{SO}_{3}\right)\left(\mathrm{CF}_{3} \mathrm{SO}_{3}\right)\right] \cdot \mathrm{CF}_{3} \mathrm{SO}_{3}\right\}(5),\left\{\left[\mathrm{Cd}\left(\mathrm{L}_{2}\right)_{2}\right.\right.$ $\left.\left.\left(\mu_{2}-\mathrm{NCS}\right)_{2}\right]\right\}_{n}(6),\left[\mathrm{Ag}\left(\mu_{2}-\mathrm{L}_{2}\right)\left(\mu_{2}-\mathrm{CF}_{3} \mathrm{SO}_{3}\right)\right]_{n}(7)$ and $\left\{\left[\mathrm{Ag}\left(\mu_{2}-\mathrm{L}_{2}\right)\right]\right.$. $\left.\mathrm{BF}_{4}\right\}_{n}(8)$ have been isolated. Both 1 and 2 are $2 \mathrm{D} \mathrm{Cd}{ }^{\mathrm{II}}$ coordination polymers containing infinite $\{\mathrm{Cd}-\mathrm{NCS}-\mathrm{Cd}\}$ chains (for $\mathbf{1}$ ) or infinite $\{\mathrm{Cd}-\mathrm{dca}-\mathrm{Cd}\}$ layers (for 2 ). 3 is a $2 \mathrm{D} \mathrm{Cu} \mathrm{Cu}^{\mathrm{II}}$ coordination polymer, in which central metal ions are bridged via bidentate bridging $\mathrm{L}_{1}$ ligand. While when different $\mathrm{Ag}^{\mathrm{I}}$ salts were introduced into the reaction system, $1 \mathrm{D}$ cluster-based $\mathrm{Ag}^{\mathrm{I}}$ coordination polymers $\mathbf{4}$ and $\mathbf{5}$ with diverse coordination modes can be isolated. Further when the isomeric oxadiazol-pyridine $L_{2}$ is used to replace $\mathrm{L}_{1}$ in the reaction system, 6-8 can be isolated. 6 is a $2 \mathrm{D} \mathrm{Cd}{ }^{\mathrm{II}}$ coordination polymer containing $2 \mathrm{D}\{\mathrm{Cd}-\mathrm{NCS}-\mathrm{Cd}\}$ layers. 7 is a $2 \mathrm{D}$ neutral $\mathrm{Ag}^{\mathrm{I}}$ coordination polymer while 8 is a $2 \mathrm{D}$ cationic $\mathrm{Ag}^{\mathrm{I}}$ coordination polymer. Variable temperature magnetic susceptibility measurements $(2-300 \mathrm{~K})$ reveal antiferromagnetic interactions between central copper(II) ions for 3. Solid-state luminescent properties of 1, $\mathbf{2}$ and 4-8 have been investigated indicating strong fluorescent emissions. Additionally, luminescent measurements illustrate that complex 8 also exhibits highly sensitive luminescence sensing for $\mathrm{Cr}_{2} \mathrm{O}_{7}{ }^{2-}$ anion pollutants in aqueous solutions with high quenching 
efficiency $K_{\mathrm{sv}}=2.08 \times 10^{4} \mathrm{~L} \mathrm{~mol}^{-1}$ and low detection limit ( 0.19 $\mu \mathrm{M}(\mathrm{S} / \mathrm{N}=3)) .8$ also represents the first report of coordination polymers based on oxadiazol-pyridine derivatives as luminescent response to $\mathrm{Cr}_{2} \mathrm{O}_{7}{ }^{2-}$ anion pollutants in the water solutions. On the basis of this work, further syntheses, structures and properties studies of these coordination polymers using these isomeric oxadiazol-pyridine derivatives as basic building blocks are also under way in our laboratory.

\section{Acknowledgements}

This work was supported financially by the Open Project of Key Lab Adv Energy Mat Chem (Nankai Univ), Tianjin Educational Committee (20120508), Natural Science Foundation of Tianjin (Grant no. 14JCQNJC05900), Young Scientist Fund (Grant no. 21301128), National Natural Science Foundation of China (Grant No. 21375095) and the Program for Innovative Research Team in University of Tianjin (TD12-5038).

\section{References}

1 (a) D. Venkataraman, S. Lee, J. S. Moore, P. Zhang, K. A. Hirsch, G. B. Gardner, A. C. Covey and C. L. Prentice, Chem. Mater., 1996, 8, 2030; (b) O. M. Yaghi, T. J. Pinnavaia and M. F. Thorpe, in Access in Nanoporous Materials, ed. Plenum, New York, 1995, p. 111; (c) A. Stein, S. W. Keller and T. E. Mallouk, Science, 1993, 259, 1558; (d) P. J. Fagan and M. D. Ward, Sci. Am., 1992, 267, 48; (e) Inclusion Compounds, ed. T. Iwamoto, J. L. Atwood, J. E. D. Davies and D. D. MacNicol, Oxford, New York, 1991, vol. 5, p. 177; (f) B. F. Hoskins and R. Robson, J. Am. Chem. Soc., 1990, 112, 1546; $(g)$ J. Lu, W. T. A. Harrison and A. J. Jacobson, Angew. Chem., Int. Ed., 1995, 34, 2557.

2 (a) B. Moulton and M.-J. Zaworotko, Chem. Rev., 2001, 101, 1629; (b) O. M. Yaghi, M. O'Keeffe, N. W. Ockwig, H. K. Chae, M. Eddaoudi and J. Kim, Nature, 2003, 423, 706; (c) S. Kitagawa, R. Kitaura and S. I. Noro, Angew. Chem., Int. Ed., 2004, 43, 2334; (d) G. Kubas, Chem. Rev., 2007, 107, 4152.

3 (a) M. Eddaoudi, J. Kim, N. Rosi, D. Vodak, J. Wachter, M. O'Keeffe and O. Yaghi, Science, 2002, 295, 469; (b) S. Bourrelly, P. Llewellyn, C. Serre, F. Millange, T. Loiseau and G. Férey, J. Am. Chem. Soc., 2005, 127, 13519; (c) B. Panella, M. Hirscher, H. Puetter and U. Mueller, $A d v$. Funct. Mater., 2006, 16, 520; (d) Y. Liu, J. F. Eubank, A. J. Cairns, J. Eckert, V. C. Kravtsov, R. Luebke and M. Eddaoudi, Angew. Chem., Int. Ed., 2007, 46, 3278; (e) A. Phan, C. J. Doonan, F. J. Uribe-Romo, C. B. Knobler, M. O'Keeffe and O. M. Yaghi, Acc. Chem. Res., 2010, 43, 58.

4 (a) R.-B. Getman, Y. S. Bae, C.-E. Wilmer and R.-Q. Snurr, Chem. Rev., 2011, 112, 703; (b) P. Horcajada, R. Gref, T. Baati, P.-K. Allan, G. Maurin, P. Couvreur, G. Férey, R.-E. Morris and C. Serre, Chem. Rev., 2011, 112, 1232; (c) L.-E. Kreno, K. Leong, O.-K. Farha, M. Allendorf, R.-P. Van Duyne and J.-T. Hupp, Chem. Rev., 2011, 112, 1105; (d) J.-R. Li, J. Sculley and H.-C. Zhou, Chem. Rev., 2011, 112, 869.
5 (a) J. R. Li, R. J. Kuppler and H. C. Zhou, Chem. Soc. Rev., 2009, 38, 1477; (b) R. Vaidhyanathan, S. S. Iremonger, G. K. H. Shimizu, P. G. Boyd, S. Alavi and T. K. Woo, Science, 2010, 330, 650; (c) P. Nugent, Y. Belmabkhout, S. D. Burd, A. J. Cairns, R. Luebke, K. Forrest, T. Pham, S. Ma, B. Space, L. Wojtas, M. Eddaoudi and M. J. Zaworotko, Nature, 2013, 495, 80; (d) H. Yin, J. Wang, Z. Xie, J. Yang, J. Bai, J. Lu, Y. Zhang, D. Yin and J. Y. S. Lin, Chem. Commun., 2014, 50, 3699.

6 (a) R. Kitaura, K. Fujimoto, S. Noro, M. Kondo and S. Kitagawa, Angew. Chem., Int. Ed., 2002, 41, 133; (b) R. Kitaura, G. Onoyama, H. Sakamoto, R. Matsuda, S. Noro and S. Kitagawa, Angew. Chem., Int. Ed., 2004, 43, 2684; (c) B. Chen, F. R. Fronczek and A. W. Maverick, Inorg. Chem., 2004, 43, 8209; (d) P. A. Maggard, B. Yan and J. Luo, Angew. Chem., Int. Ed., 2005, 44, 2; (e) R. Custelcean and M. G. Gorbunova, J. Am. Chem. Soc., 2005, 127, 16362; (f) Y. Yoshida, K. Inoue and M. Kurmoo, Inorg. Chem., 2009, 48, 267; $(g)$ Y. Inokuma, T. Arai and M. Fujita, Nat. Chem., 2010, 2, 780; (h) M. J. Manos, E. J. Kyprianidou, G. S. Papaefstathiou and A. J. Tasiopoulos, Inorg. Chem., 2012, 51, 6308.

7 (a) M. Kurmoo, Chem. Soc. Rev., 2009, 38, 1353; (b) P. Mahata, S. Natarajan, P. Panissod and M. Drillon, J. Am. Chem. Soc., 2009, 131, 10140; (c) G. Rogez, N. Virat and M. Drillon, Angew. Chem., Int. Ed., 2010, 49, 1921; (d) D. D. Sante, A. Stroppa, P. Jain and S. Picozzi, J. Am. Chem. Soc., 2013, 135, 18126; (e) A. Stroppa, P. Barone, P. Jain, J. M. PerezMato and S. Picozzi, Adv. Mater., 2013, 25, 2284; $(f)$ G. Beobide, W. Wang, O. Castillo, A. Luque, P. Román, G. Tagliabue, S. Galli and J. A. R. Navarro, Inorg. Chem., 2008, 5267.

8 (a) Y. Takashima, V. M. Martinez, S. Furukawa, M. Kondo, S. Shimomura, H. Uehara, M. Nakahama, K. Sugimoto and S. Kitagawa, Nat. Commun., 2011, 2, 168; (b) D. N. Dybtsev, H. Chun and K. Kim, Angew. Chem., Int. Ed., 2004, 43, 5033. 9 (a) E. Y. Lee and M. P. Suh, Angew. Chem., Int. Ed., 2004, 43, 2798; (b) C. L. Chen, A. M. Goforth, M. D. Smith, C. Y. Su and H. C. zur Loye, Angew. Chem., Int. Ed., 2005, 44, 6673; (c) Q. Chu, D. C. Swenson and L. R. MacGillivray, Angew. Chem., Int. Ed., 2005, 44, 3569; (d) J. P. Ma, Y. B. Dong, R. Q. Huang, M. D. Smith and C. Y. Su, Inorg. Chem., 2005, 44, 6143; (e) J. Y. Lee, S. Y. Lee, W. Sim, K. M. Park, J. Kim and S. S. Lee, J. Am. Chem. Soc., 2008, 130, 6902; (f) Z. Wang and S. M. Cohen, J. Am. Chem. Soc., 2007, 129, 12368.

10 (a) Y. Han, X. Li, L. Li, C. Ma, Z. Shen, Y. Song and X. You, Inorg. Chem., 2010, 49, 10781; (b) S. Dang, J.-H. Zhang, Z.-M. Sun and H. Zhang, Chem. Commun., 2012, 48, 11139; (c) S. Dang, J.-H. Zhang and Z.-M. Sun, J. Mater. Chem., 2012, 22, 8868; (d) W.-H. Zhu, Z.-M. Wang and S. Gao, Inorg. Chem., 2007, 46, 1337.

11 (a) J. Rocha, L. D. Carlos, F. A. A. Paz and D. Ananias, Chem. Soc. Rev., 2011, 40, 926; (b) D. Banerjee, Z. Hu and J. Li, Dalton Trans., 2014, 43, 10668; (c) L. Zhang, Z. Kang, X. Xin and D. Sun, CrystEngComm, 2016, 18, 193; (d) D. Zhao, Y. Cui, Y. Yang and G. Qian, CrystEngComm, 2016, 18, 3746. 
12 (a) D. J. Tranchemontagne, J. L. Mendoza-Cortés, M. O'Keeffe and O. M. Yaghi, Chem. Soc. Rev., 2009, 38, 1257; (b) L. Ma, C. Abney and W. Lin, Chem. Soc. Rev., 2009, 38, 1248; (c) J. Lee, O. K. Farha, J. Roberts, K. A. Scheidt, S. T. Nguyen and J. T. Hupp, Chem. Soc. Rev., 2009, 38, 1450; (d) M. D. Allendorf, C. A. Bauer, R. K. Bhakta and R. J. T. Houk, Chem. Soc. Rev., 2009, 38, 1330.

13 (a) H. Kajiro, A. Kondo, K. Kaneko and H. Kanoh, Int. J. Mol. Sci., 2010, 11, 3803; (b) L. Carlucci, G. Ciani, D. M. Proserpio, T. G. Mitina and V. A. Blatov, Chem. Rev., 2014, 114, 7557; (c) A. Schneemann, V. Bon, I. Schwedler, I. Senkovska, S. Kaskel and R. A. Fischer, Chem. Soc. Rev., 2014, 43, 6062.

14 (a) R. Kitaura, K. Seki, G. Akiyama and S. Kitagawa, Angew. Chem., Int. Ed., 2003, 42, 428; (b) A. Kondo, H. Noguchi, S. Ohnishi, H. Kajiro, A. Tohdoh, Y. Hattori, W. C. Xu, H. Tanaka, H. Kanoh and K. Kaneko, Nano Lett., 2006, 6, 2581; (c) A. Kondo, T. Nakagawa, H. Kajiro, A. Chinen, Y. Hattori, F. Okino, T. Ohba, K. Kaneko and H. Kanoh, Inorg. Chem., 2010, 49, 9247; (d) D. Xiao, H. Chen, D. Sun, J. He, S. Yan, J. Yang, X. Wang, R. Yuan and E. Wang, CrystEngComm, 2012, 14, 2849; (e) A. Kondo, N. Kojima, H. Kajiro, H. Noguchi, Y. Hattori, F. Okino, K. Maeda, T. Ohba, K. Kaneko and H. Kanoh, J. Phys. Chem. C, 2012, 116, 4157; $(f)$ Y. Jiang, J. Huang, B. Kasumaj, G. Jeschke, M. Hunger, T. Mallat and A. Baiker, J. Am. Chem. Soc., 2009, 131, 2058.

15 (a) Y. B. Dong, Q. Zhang, L. L. Liu, J.-P. Ma, B. Tang and R. Q. Huang, J. Am. Chem. Soc., 2007, 129, 1514; (b) Y. F. Han, W. G. Jia, Y. J. Lin and G. X. Jin, Angew. Chem., Int. Ed., 2009, 34, 6234.

16 (a) J.-G. Haasnoot, Coord. Chem. Rev., 2000, 200-202, 131; (b) J. P. Zhang, S. Horike and S. Kitagawa, Angew. Chem., Int. Ed., 2007, 46, 889.

17 (a) C. H. Lee, H. Y. Huang, Y. H. Liu, T. T. Luo, G. H. Lee, S. M. Peng, J. C. Jiang, I. Chao and K. L. Lu, Inorg. Chem., 2013, 52, 3962; (b) G.-M. Sun, F. Luo, Y.-M. Song, X.-Z. Tian, H.-X. Huang, Y. Zhu, Z.-J. Yuan, X.-F. Feng, M.-B. Luo, S.-J. Liu and W.-Y. Xu, Dalton Trans., 2012, 41, 11559.

18 B.-C. Tzeng and T.-Y. Chang, Cryst. Growth Des., 2009, 9, 5343.

19 Y. B. Dong, T. Sun, J. P. Ma, X. X. Zhao and R. Q. Huang, Inorg. Chem., 2006, 45, 10613.

20 J.-P. Ma, C.-W. Zhao, S.-Q. Wang, J.-P. Zhang, X. Niu and Y.-B. Dong, Chem. Commun., 2015, 51, 14586.

21 (a) B. Ding, L. Yi, Y. Wang, P. Cheng, D.-Z. Liao, S.-P. Yan and Z.-H. Jiang, Dalton Trans., 2006, 665; (b) B. Ding, L. Yi, P. Cheng, D. Z. Liao and S. P. Yan, Inorg. Chem., 2006, 45, 5799; (c) B. Ding, Y.-Y. Liu, Y. Q. Huang, W. Shi, P. Cheng, D.-Z. Liao and S.-P. Yan, Cryst. Growth Des., 2009, 9, 593; (d) E.-C. Yang, H.-K. Zhao, B. Ding, X.-G. Wang and X.-J. Zhao, Cryst. Growth Des., 2007, 7, 2009; (e) B. Ding, P. Yang, Y.-Y. Liu, Y. Wang and G.-X. Du, CrystEngComm, 2013, 15, 2490; (f) X.-X. Wu, Y.-Y. Wang, P. Yang, Y.-Y. Xu, J.-Z. Huo, B. Ding, Y. Wang and X.-G. Wang, Cryst. Growth Des., 2014, 14, 477; $(g)$ J. Y. Liu, Q. Wang, L. J. Zhang,
B. Yuan, Y. Y. Xu, X. Zhang, C. Y. Zhao, D. Wang, Y. Yuan, Y. Wang, B. Ding, X. J. Zhao and M. M. Yue, Inorg. Chem., 2014, 53, 5972; (h) Y. Wang, B. Yuan, Y. Y. Xu, X. G. Wang, B. Ding and X. J. Zhao, Chem.-Eur. J., 2015, 21, 2107.

22 G. Navarrete-Vázqueza, G. M. Molina-Salinasb, Z. V. DuarteFajardoa, J. Vargas-Villarrealb, S. Estrada-Sotoa, F. GonzálezSalazarb, E. Hernández-Núñeza and S. Said-Fernándezb, Bioorg. Med. Chem., 2009, 46, 309.

23 G. M. Sheldrick, SHELXS-97, Program for X-ray Crystal Structure Solution, Göttingen University, Göttingen, Germany, 1997.

24 G. M. Sheldrick, SHELXL-97, Program for X-ray Crystal Structure Refinement, Göttingen University, Göttingen, Germany, 1997.

25 (a) B. Salignac, S. Riedel, A. Dolbecq, F. Sécheresse and E. Cadot, J. Am. Chem. Soc., 2000, 122, 10381; (b) A. Hijazi, J. C. Kemmegne-Mbouguen, S. Floquet, J. Marrot, J. Fize, V. Artero and E. Cadot, Dalton Trans., 2013, 42, 4848.

26 (a) A. Barba-Bon, A. M. Costero, S. Gil, M. Parra, J. Soto, R. Martinez-Manezac and F. Sancenon, Chem. Commun., 2012, 48, 3000; (b) G. S. Yang, M. N. Li, S. L. Li, Y. Q. Lan, W. W. He, X. L. Wang, J. S. Qin and Z. M. Su, J. Mater. Chem., 2012, 22, 17947.

27 Z. G. Gu, Y. F. Xu, X. H. Zhou, J. L. Zuo and X. Z. You, Cryst. Growth Des., 2008, 8, 1306.

28 (a) Z. Wang, K. Hu, S. Gao and H. Kobayashi, Adv. Mater., 2010, 22, 1526; (b) G. Rogez, N. Viart and M. Drillon, Angew. Chem., Int. Ed., 2010, 49, 1921; (c) D. Di Sante, A. Stroppa, P. Jain and S. Picozzi, J. Am. Chem. Soc., 2013, 135, 18126; (d) M. Mączka, A. Ciupa, A. Gągor, A. Sieradzki, A. Pikul, B. Macalik and M. Drozd, Inorg. Chem., 2014, 53, 5260.

29 (a) X. Li, X. W. Wang and Y. H. Zhang, Inorg. Chem. Commun., 2008, 11, 832; (b) C. A. Bauer, T. V. Timofeeva, T. B. Settersten, B. D. Patterson, V. H. Liu, B. A. Simmons and M. D. Allendorf, J. Am. Chem. Soc., 2007, 129, 7136.

30 (a) S. H. Rahaman, R. Ghosh, G. Mostafa and B. K. Ghosh, Inorg. Chem. Commun., 2005, 8, 1137; (b) Y. Lui and R. T. Tiekink, CrystEngComm, 2005, 7, 20; (c) G. Singha, S. Girdhara and M. Garga, Synth. React. Inorg., Met.-Org., Nano-Met. Chem., 2013, 43, 1107-1111.

31 (a) J. M. Hao, B. Y. Yu, K. V. Hecke and G. H. Cui, CrystEngComm, 2015, 17, 2279; (b) G. H. Cui, C. H. He, C. H. Jiao, J. C. Geng and V. A. Blatov, CrystEngComm, 2012, 14, 4210; (c) X. X. Wang, B. Y. Yu, K. V. Hecke and G. H. Cui, $R S C A d v$., 2014, 4, 61281; (d) L. Qin, J. Zheng, S. L. Xiao, X. H. Zheng and G. H. Cui, Inorg. Chem. Commun., 2013, 34, 71; (e) X. X. Wang, M. X. Zhang, B. Y. Yu, K. V. Hecke and G. H. Cui, Spectrochim. Acta, Part A, 2015, 139, 442.

32 (a) C. M. Che, C. W. Wan, K. Y. Ho and Z. Y. Zhou, New J. Chem., 2001, 25, 63; (b) J. H. Luo, M. C. Hong, R. H. Wang, R. Cao, L. Han and Z. Z. Lin, Eur. J. Inorg. Chem., 2003, 2705; (c) X. L. Wang, C. Qin, E. B. Wang and Z. M. Su, Chem.-Eur. J., 2006, 12, 2680.

33 (a) C. X. Chen, Q. K. Liu, J. P. Ma and Y. B. Dong, J. Mater. Chem., 2012, 22, 9027; (b) C. Fang, Q. K. Liu, J. P. Ma and Y. B. Dong, Inorg. Chem., 2012, 51, 3923.

34 (a) Q. G. Zhai, X. Y. Wu, S. M. Chen, C. Z. Lu and W. B. Yang, Cryst. Growth Des., 2006, 6, 2126; (b) C. Ren, Y. N. Zhang, 
W. J. Shi, B. Liu, Y. Y. Wang and Q. Z. Shi, CrystEngComm, 2011, 13, 5179; (c) J. Y. Gao, N. Wang, X. H. Xiong, C. J. Chen, W. P. Xie, X. R. Ran, Y. Long, S. T. Yue and Y. L. Liu, CrystEngComm, 2013, 15, 3261.

35 K. L. Wong, G. L. Law, Y. Y. Yang and W. T. Wong, Adv. Mater., 2006, 18, 1051.

36 (a) C. X. Chen, Q. K. Liu, J. P. Ma and Y. B. Dong, J. Mater. Chem., 2012, 22, 9027; (b) C. Fang, Q. K. Liu, J. P. Ma and Y. B. Dong, Inorg. Chem., 2012, 51, 3923.

37 (a) Q. G. Zhai, X. Y. Wu, S. M. Chen, C. Z. Lu and W. B. Yang, Cryst. Growth Des., 2006, 6, 2126; (b) C. Ren, Y. N. Zhang, W. J. Shi, B. Liu, Y. Y. Wang and Q. Z. Shi, CrystEngComm, 2011, 13, 5179; (c) J. Y. Gao, N. Wang, X. H. Xiong, C. J. Chen, W. P. Xie, X. R. Ran, Y. Long, S. T. Yue and Y. L. Liu, CrystEngComm, 2013, 15, 3261.
38 P. F. Shi, B. Zhao, G. Xiong, Y. L. Hou and P. Cheng, Chem. Commun., 2012, 48, 8231.

39 K. L. Wong, G. L. Law, Y. Y. Yang and W. T. Wong, Adv. Mater., 2006, 18, 1051.

40 Z. Xie, L. Ma, K. E. deKrafft, A. Jin and W. Lin, J. Am. Chem. Soc., 2010, 132, 922.

41 (a) W. S. Liu, T. Q. Jiao, Y. Z. Li, Q. Z. Liu, M. Y. Tan, H. Wang and L. F. Wang, J. Am. Chem. Soc., 2004, 126, 2280; (b) X. Yang, Z. Huang, J. Dang, C. L. Ho, G. Zhou and W. Y. Wong, Chem. Commun., 2013, 49, 4406; (c) H. X. Zhao, L. Q. Liu, Z. D. Liu, Y. Wang, X. J. Zhao and C. Z. Huang, Chem. Commun., 2011, 47, 2604.

42 (a) Z. C. Hu, B. J. Deibert and J. Li, Chem. Soc. Rev., 2014, 43, 5815; (b) H. Xu, C. S. Cao, X. M. Kang and B. Zhao, Dalton Trans., 2016, 45, 18003. 